Supporting Information 2

$$
{ }^{1} \mathrm{H} \text { and }{ }^{13} \mathrm{C} \text { NMR }
$$

Total Synthesis of ( \pm )-Taiwaniaquinol B via a Domino Intramolecular Friedel-Crafts Acylation/Carbonyl $\alpha$ tert-Alkylation Reaction

\author{
Eric Fillion* and Dan Fishlock
}

Department of Chemistry, University of Waterloo, Waterloo, Ontario N2L 3G1, Canada

efillion@uwaterloo.ca 
Methyl 4-bromo-3,5-methoxybenzoate (6)

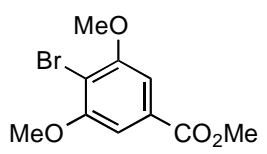

df5-185h
proton, 16 scans AVANCE-300B

홈
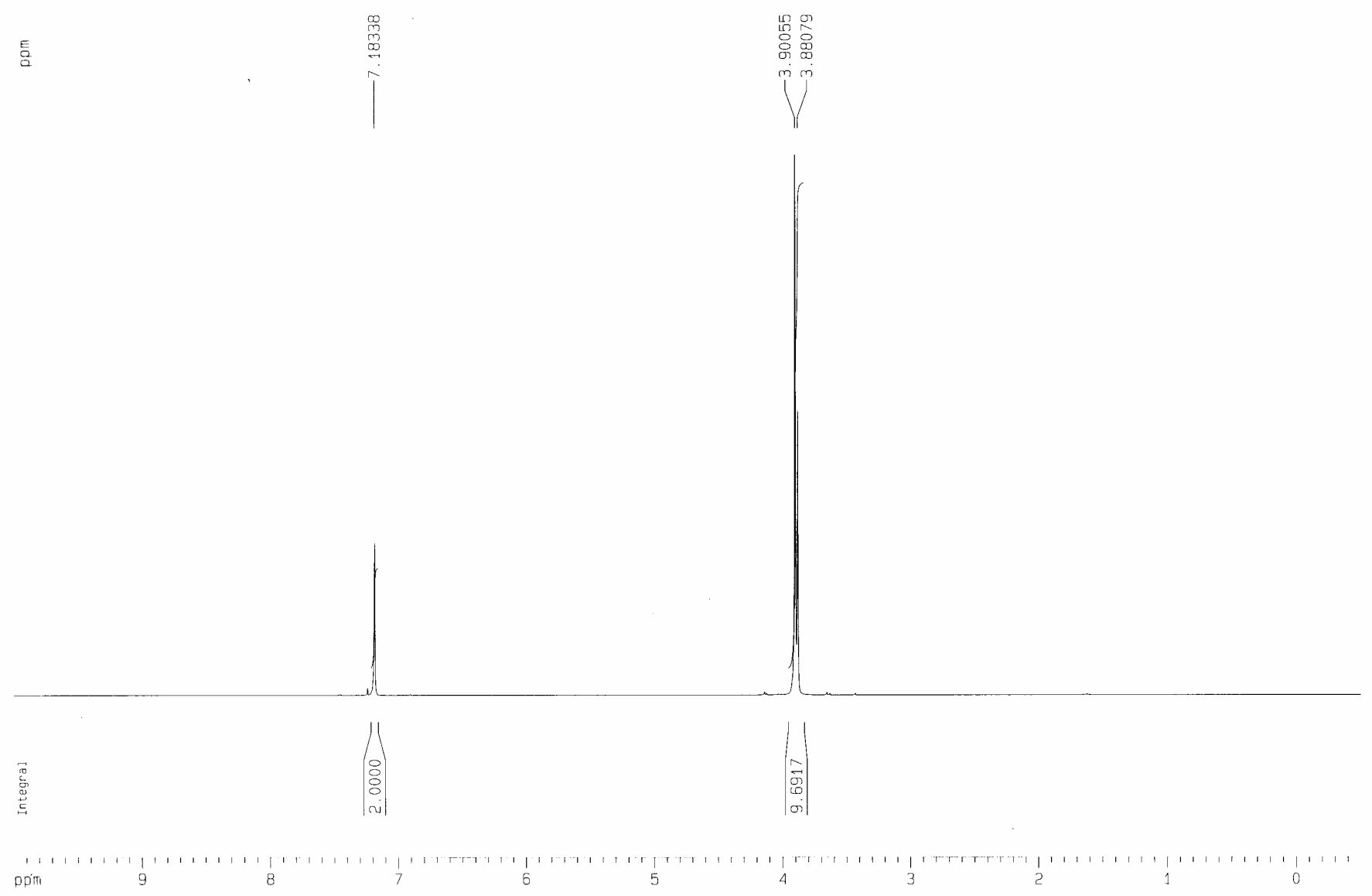

SI-2-2 
Methyl 4-bromo-3,5-methoxybenzoate (6)

C-13 with Decoupling AVANCE-300B

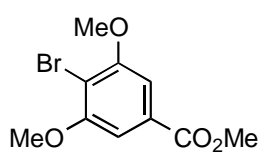

言

|

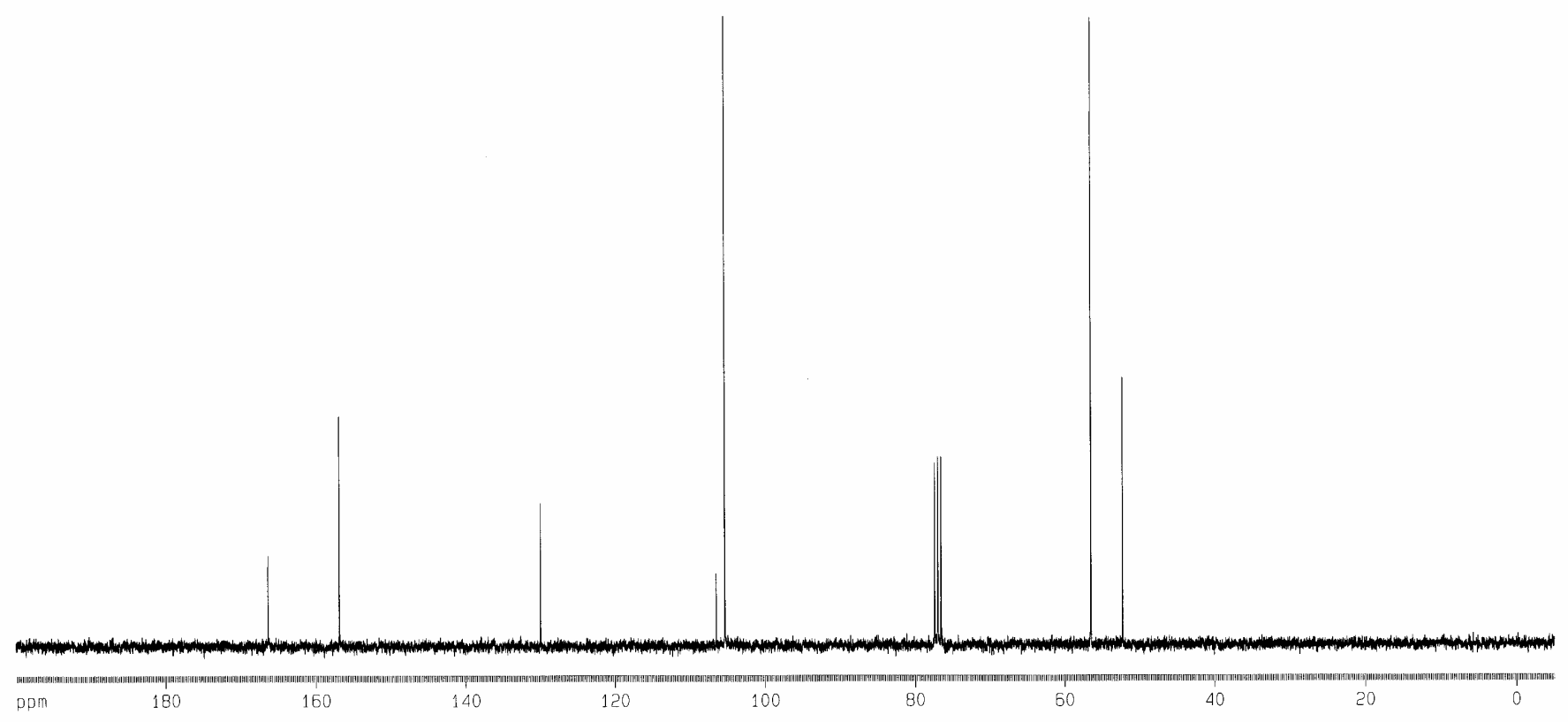


Methyl 4-isoprenyl-3,5-dimethoxybenzoate (7)

df5-152h

benzened6
proton, 16 scans

言
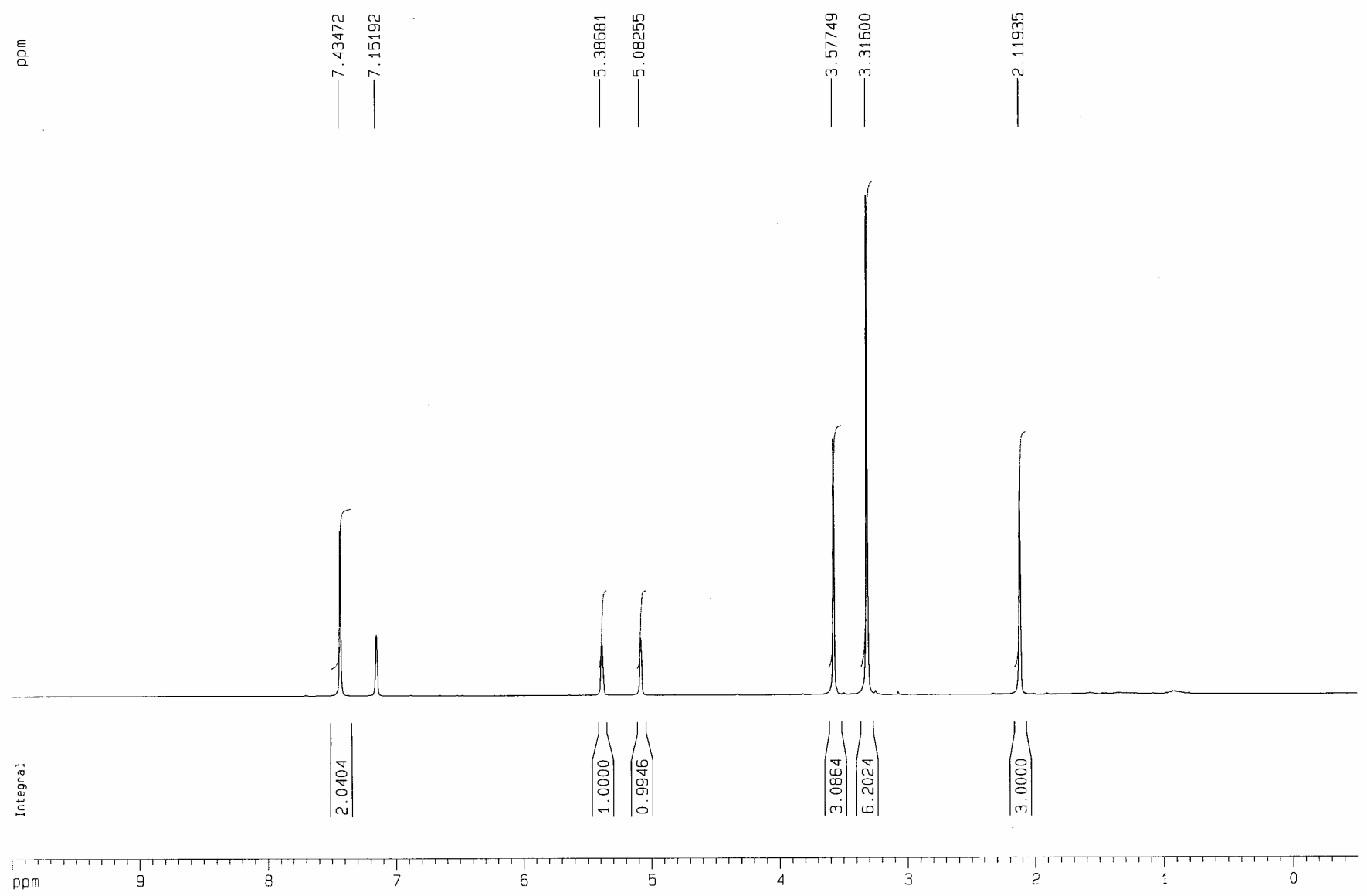

SI-2-4 
Methyl 4-isoprenyl-3,5-dimethoxybenzoate (7)

df $5-152 c 13$

$13 \mathrm{C}$ observe with $1 \mathrm{H}$ decoupling

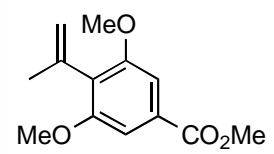

言
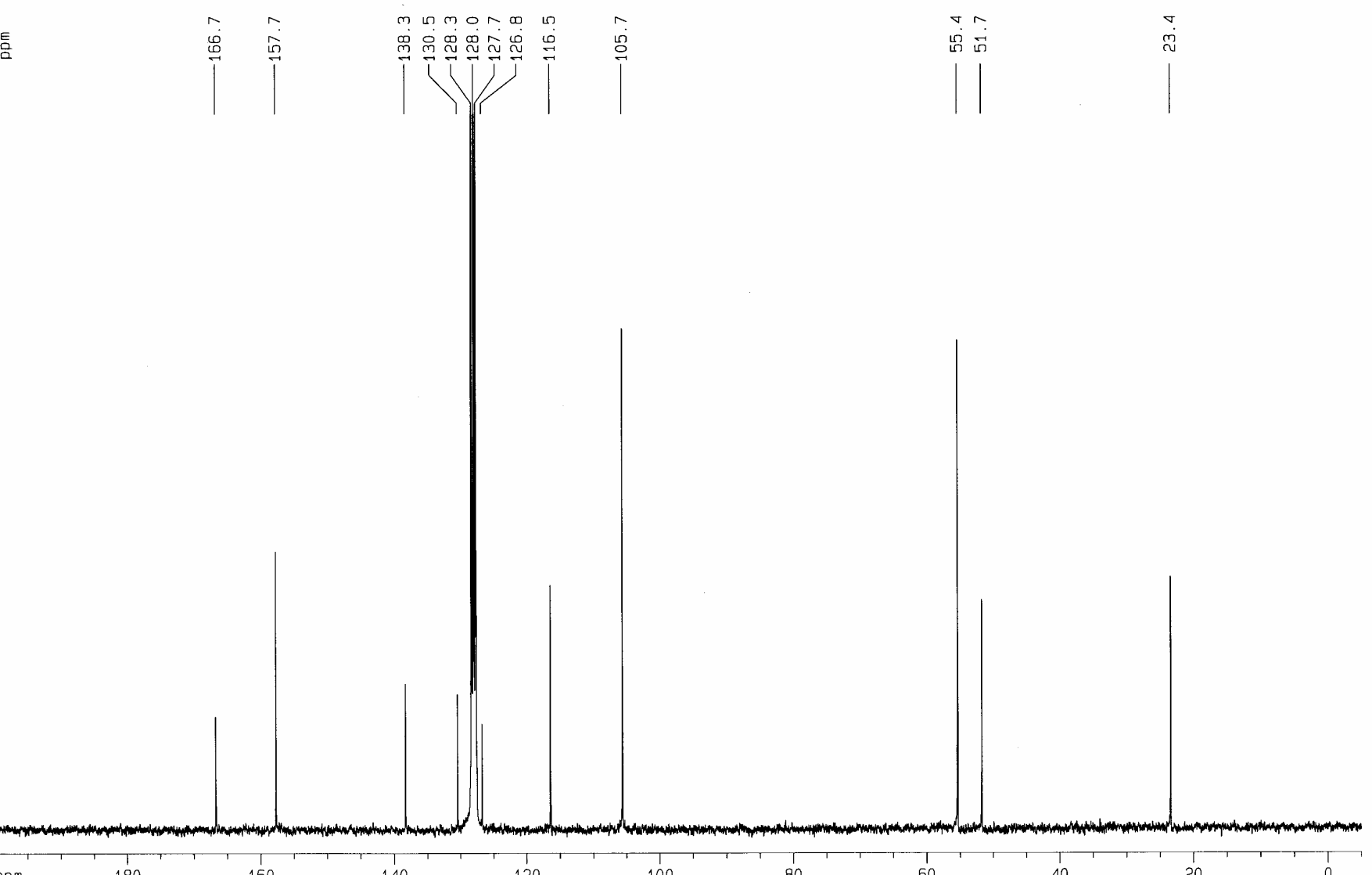
4-Isopropyl-3,5-dimethoxybenzoic acid

df5-155h 1

proton, 16 scans
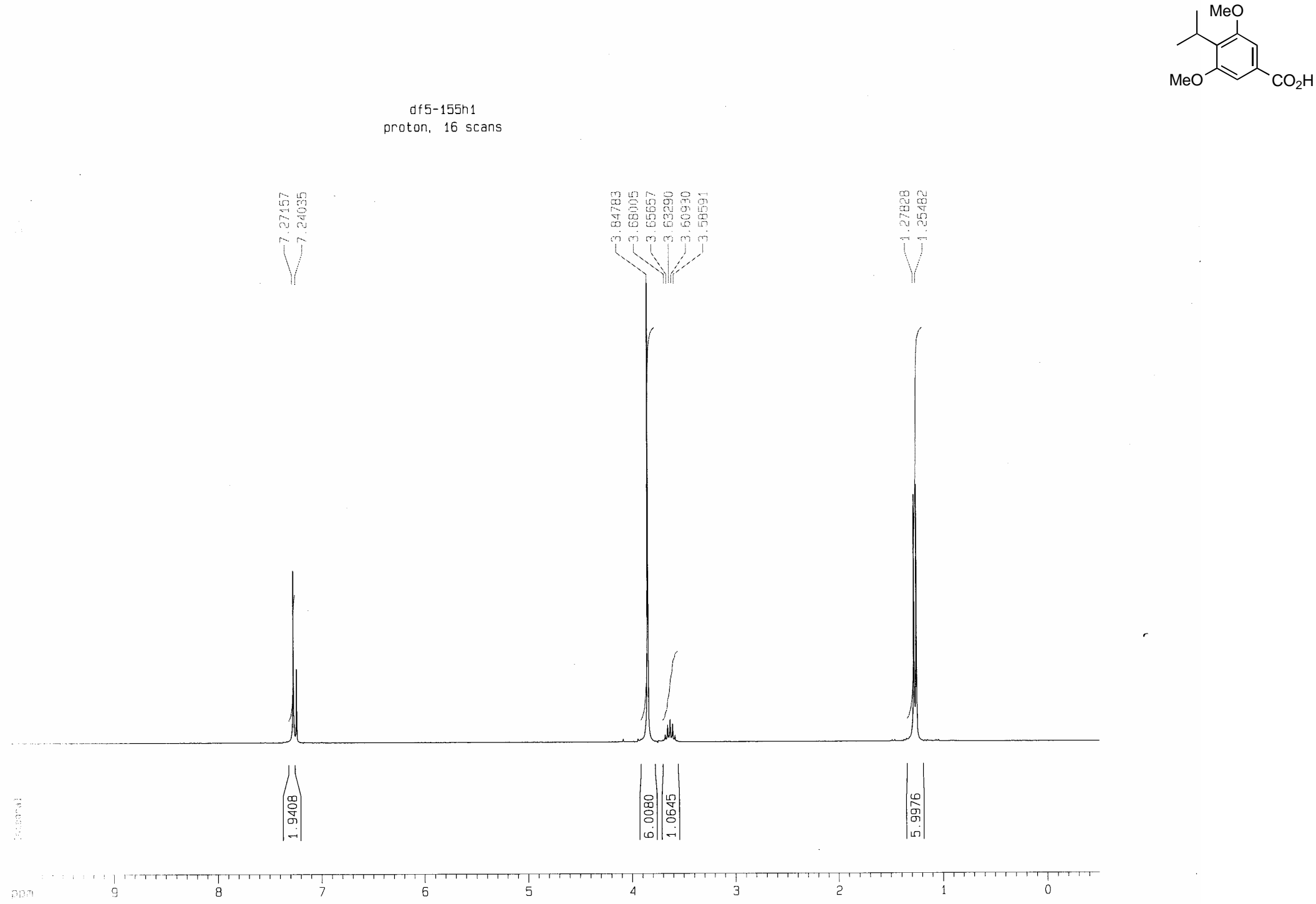
4-Isopropyl-3,5-dimethoxybenzoic acid

df5-155c 13

13C observe with $1 \mathrm{H}$ decoupling

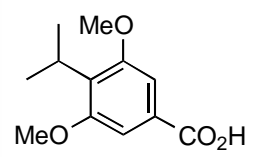

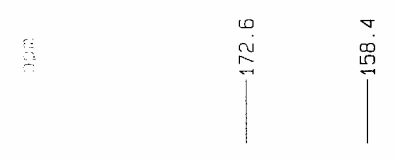
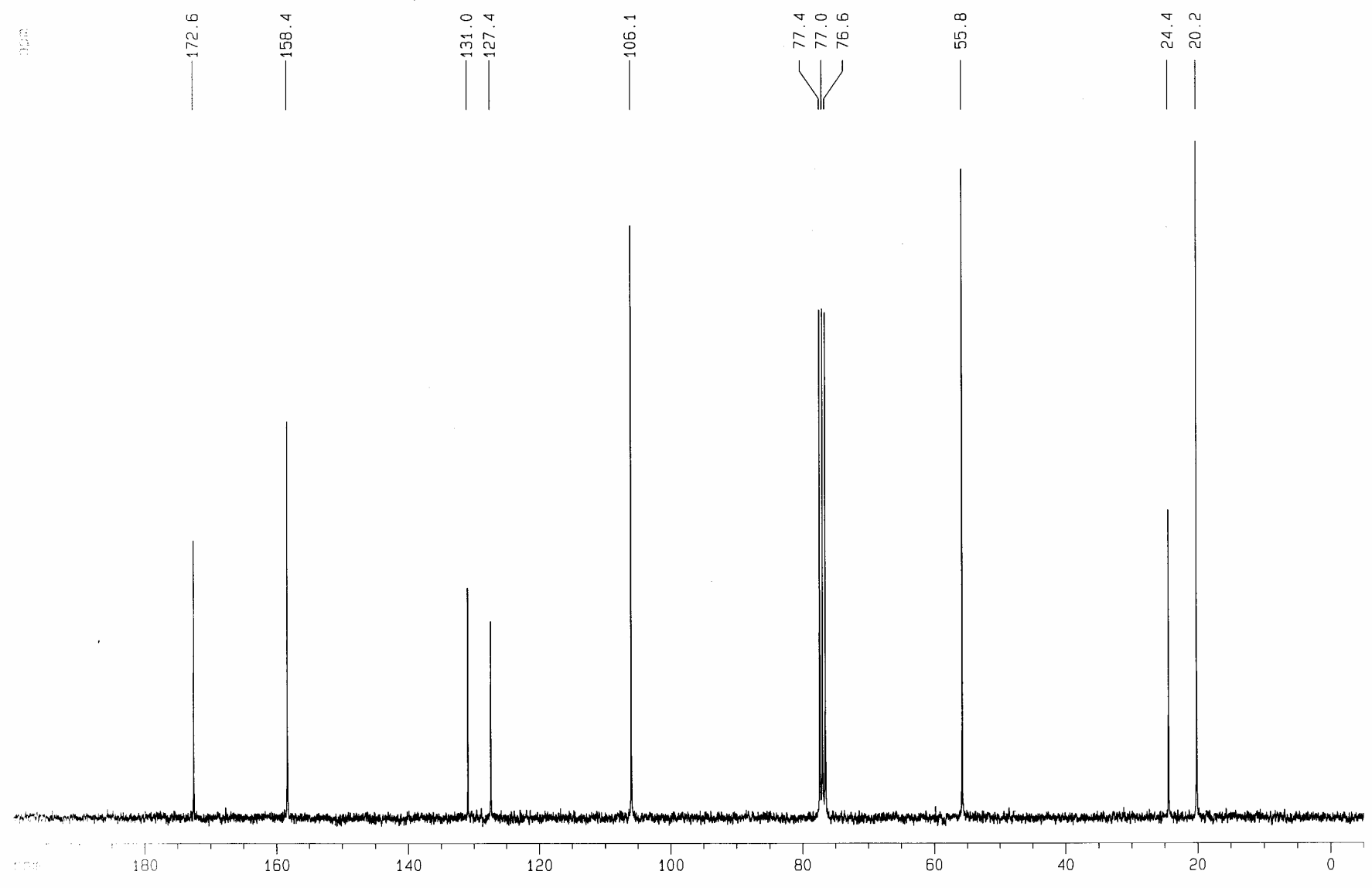

SI-2-7 
4-Isopropyl-3,5-dimethoxyacetophenone (8)

df $5-173 \mathrm{~h}$

proton, 16 scans

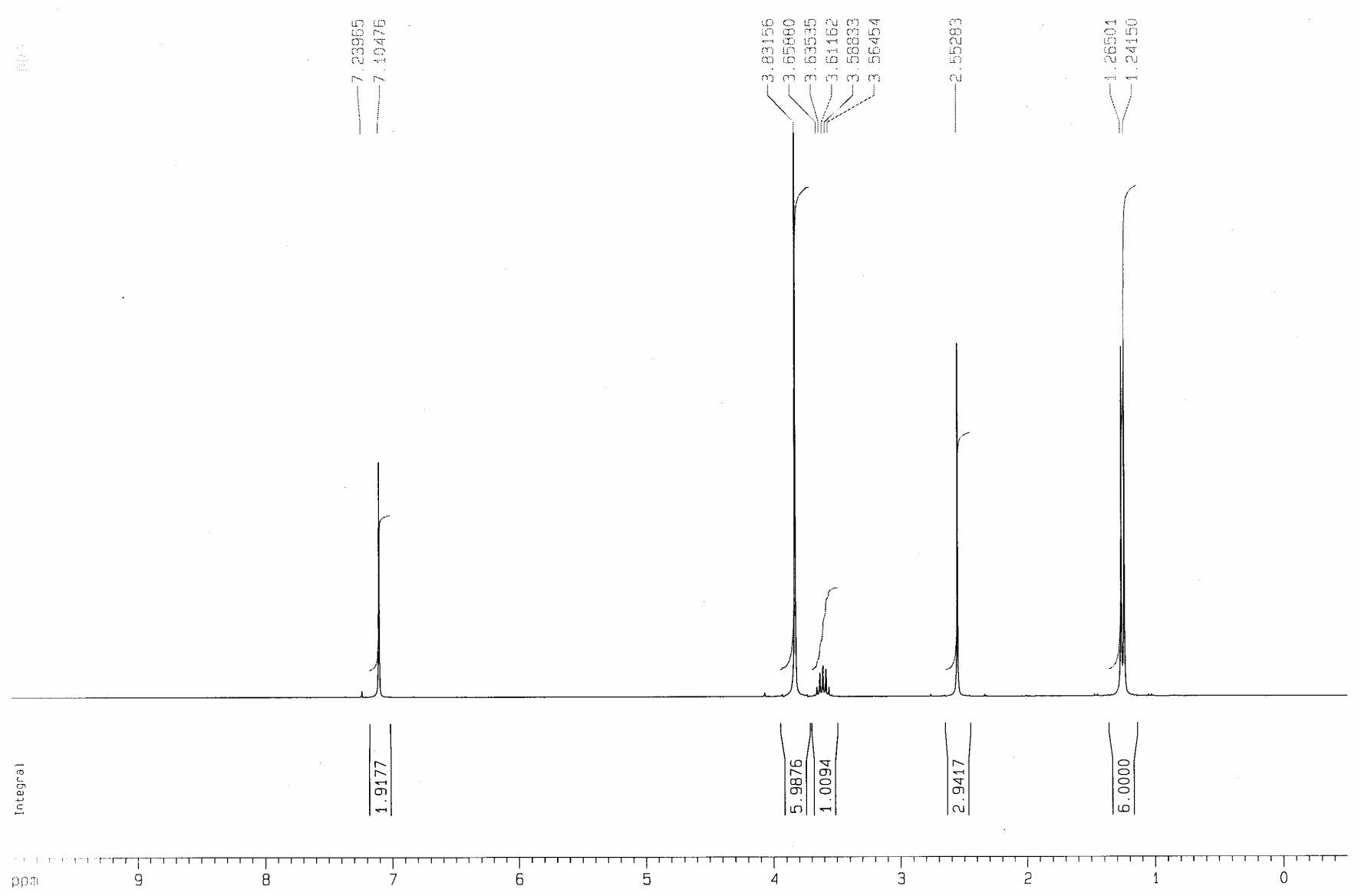


4-Isopropyl-3,5-dimethoxyacetophenone (8)

df $5-173 c 13$

$13 \mathrm{C}$ observe with $1 \mathrm{H}$ decoupling

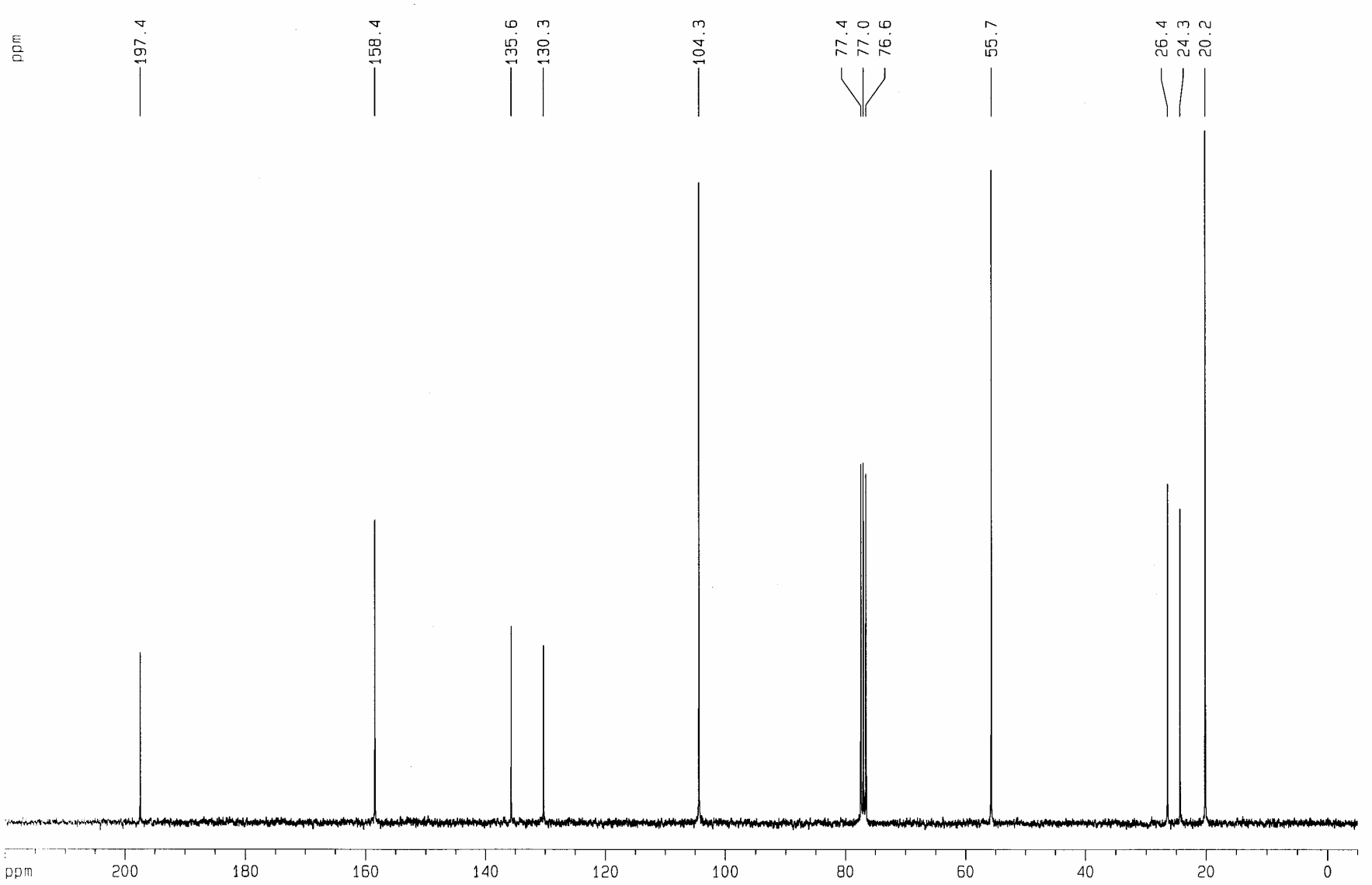


df5-176h1

proton, 16 scans
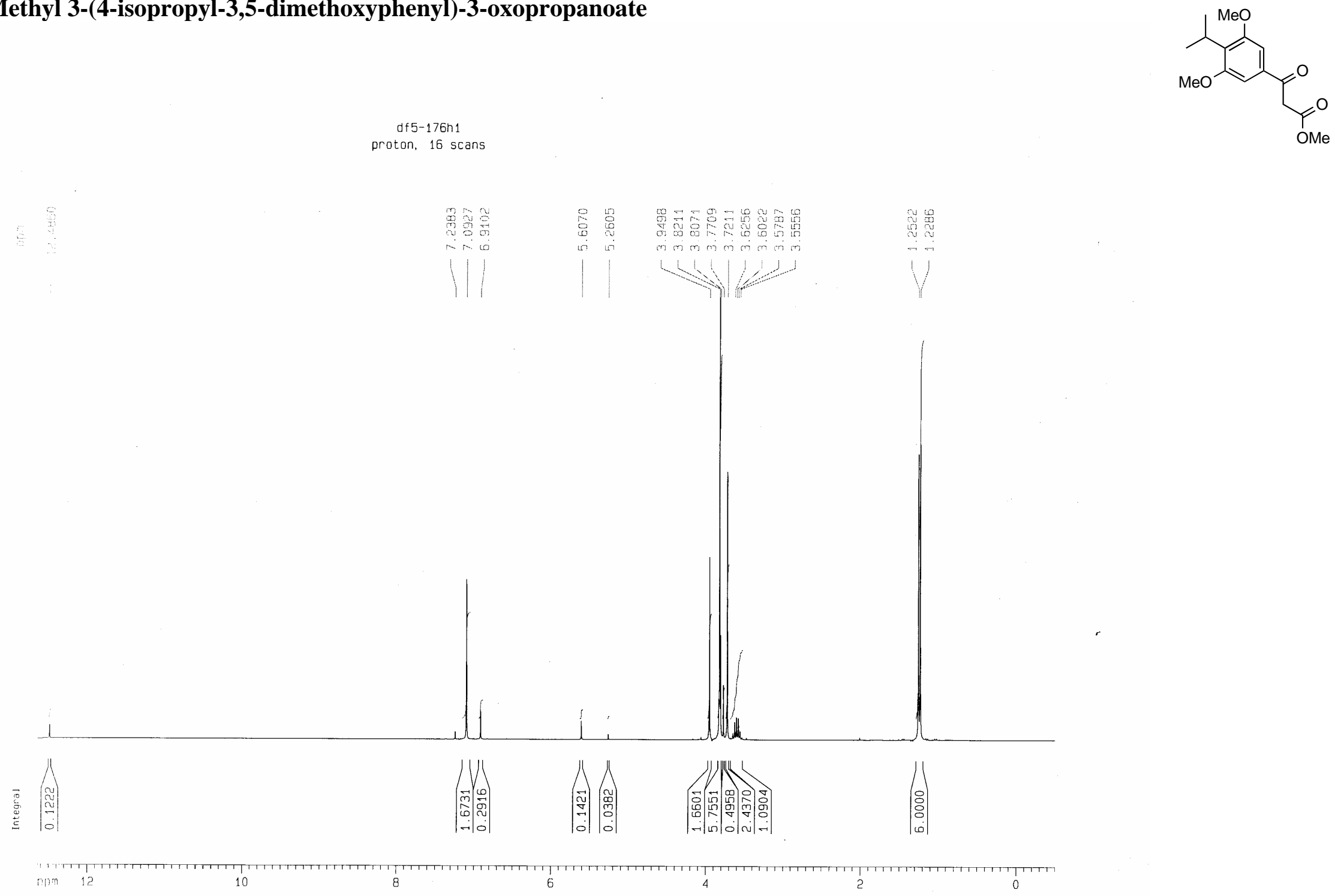
Methyl 3-(4-isopropyl-3,5-dimethoxyphenyl)-3-oxopropanoate

$13 \mathrm{C}$ observe with $1 \mathrm{H}$ decoupling
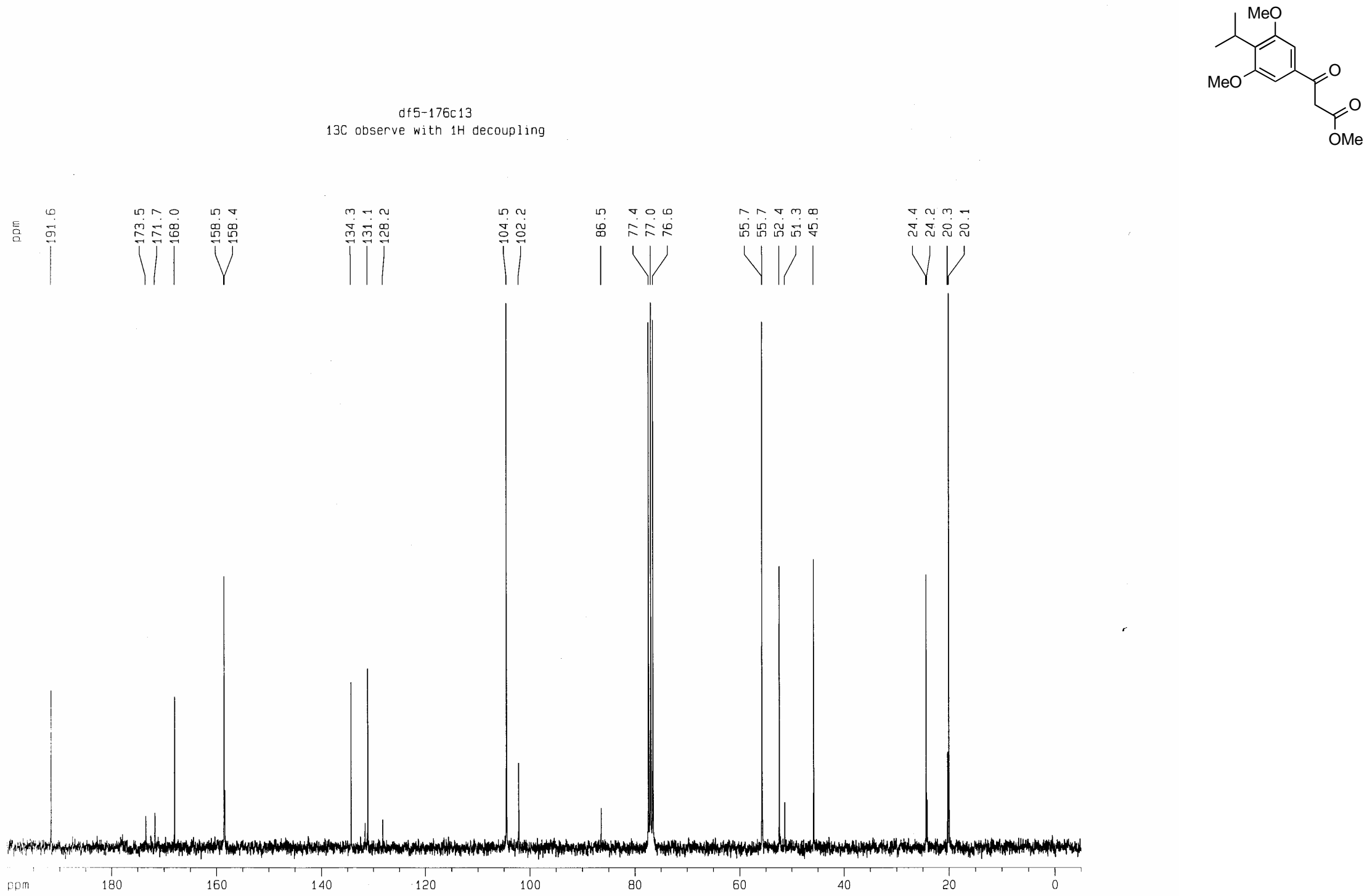

SI-2-11 


\section{1-(4-Isopropyl-3,5-dimethoxyphenyl)-5-methyl-5-hexen-1-one (9)}

df5-179bh1 proton, 16 scans
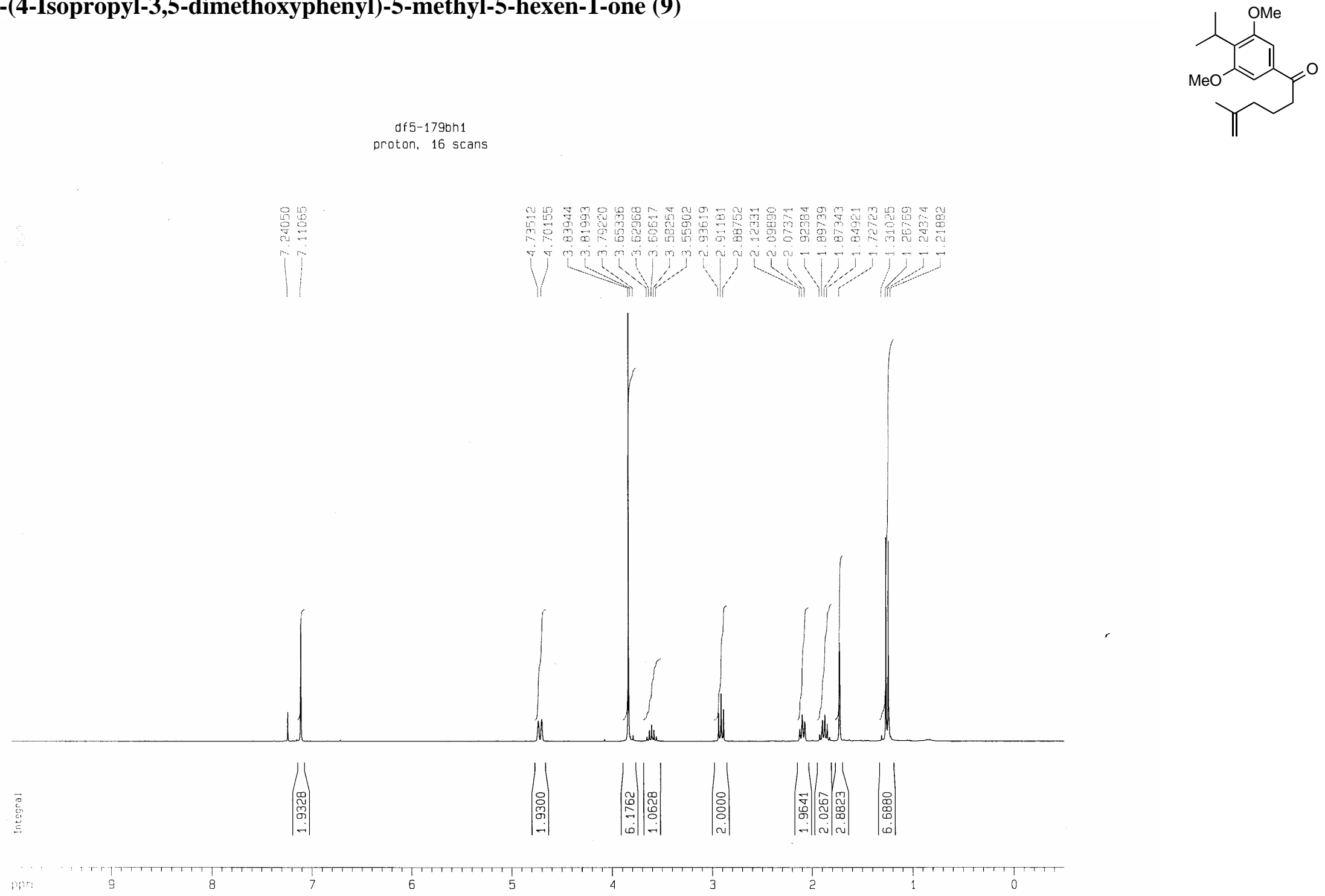

SI-2-12 


\section{1-(4-Isopropyl-3,5-dimethoxyphenyl)-5-methyl-5-hexen-1-one (9)}

df 5-179bc 13

$13 \mathrm{C}$ observe with $1 \mathrm{H}$ decoupling
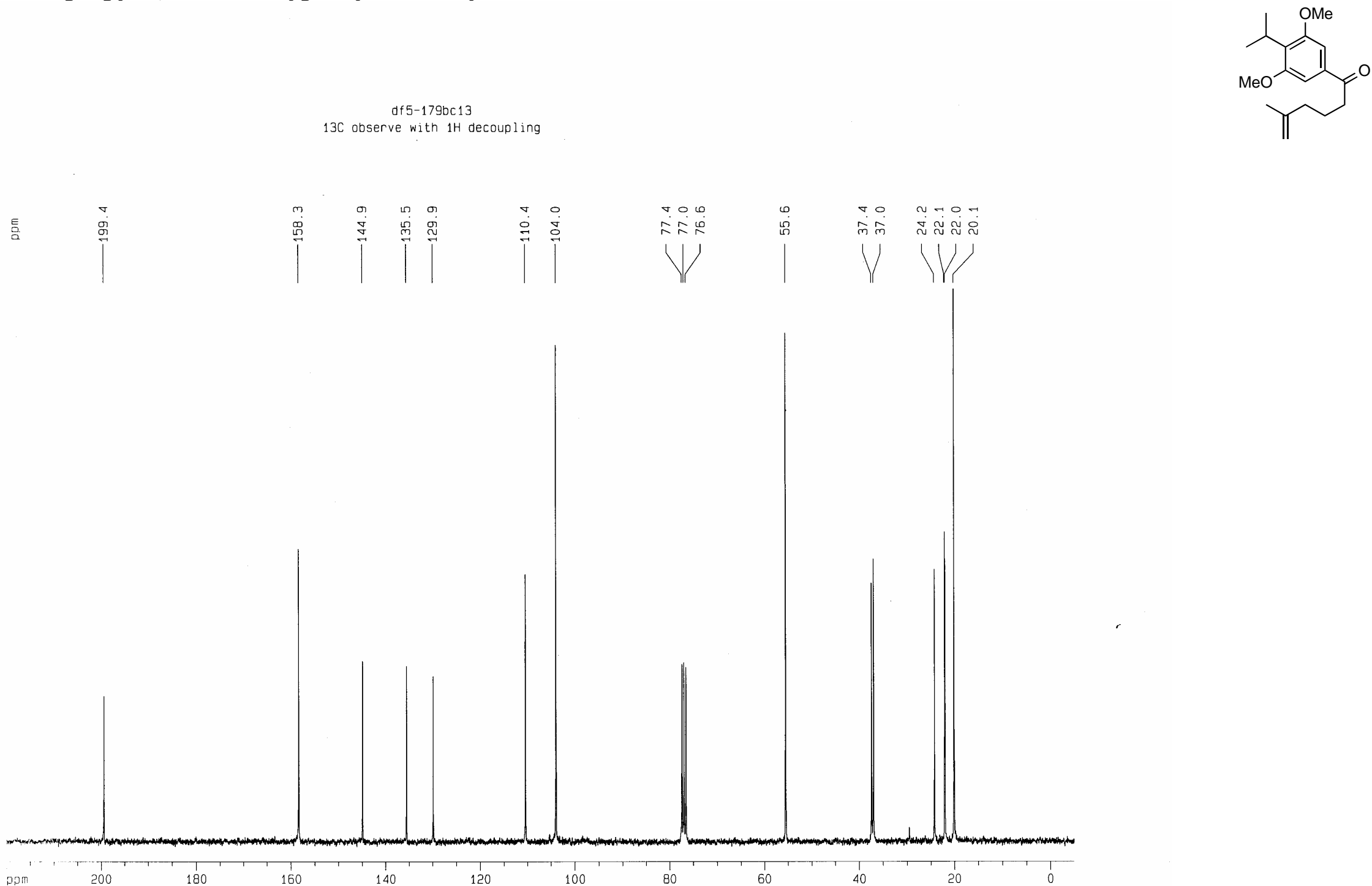

SI-2-13 
df $-182 h 1$ proton, 16 scans
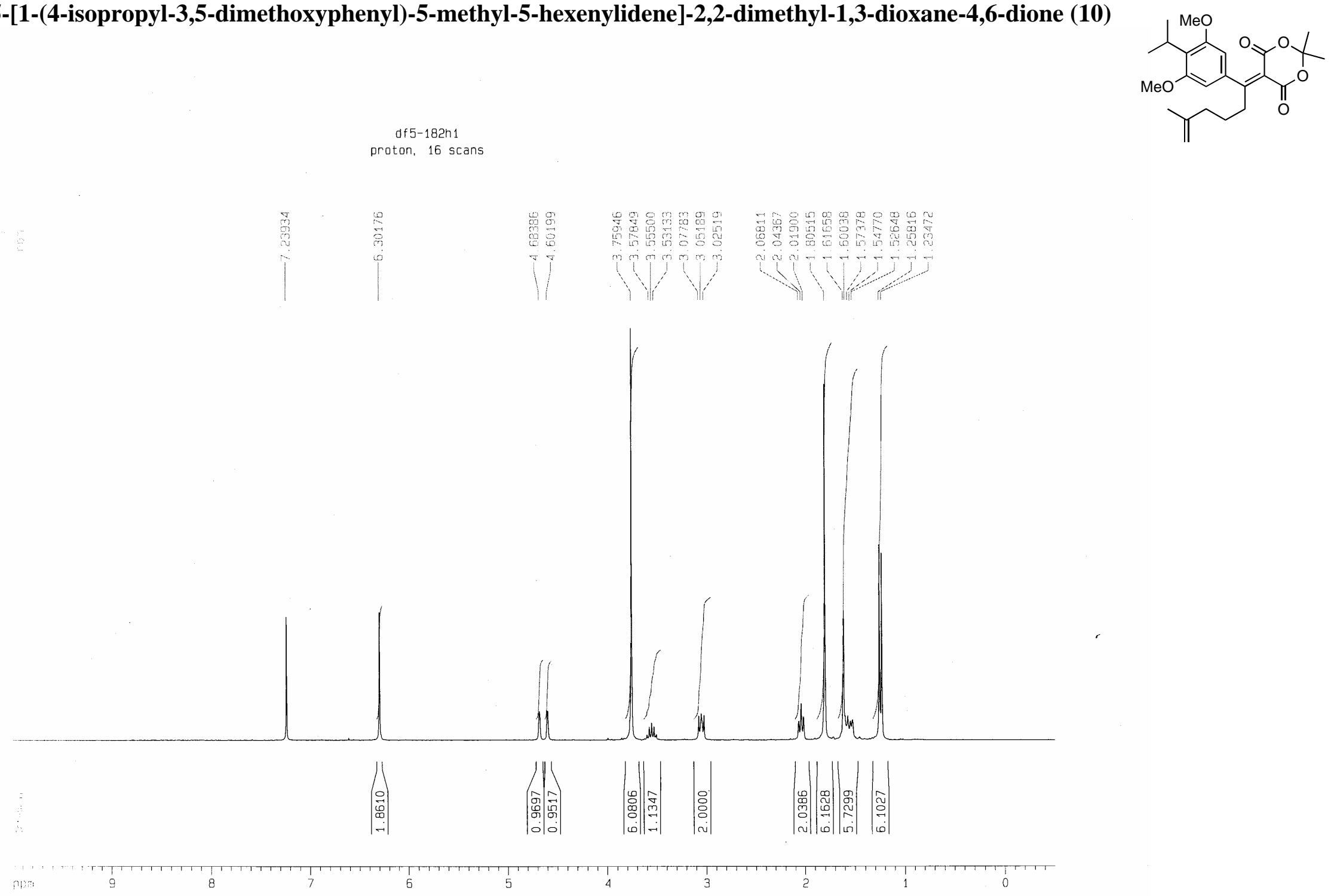
5-[1-(4-isopropyl-3,5-dimethoxyphenyl)-5-methyl-5-hexenylidene]-2,2-dimethyl-1,3-dioxane-4,6-dione (10)

df5-182c13

with $1 H$ decoupling
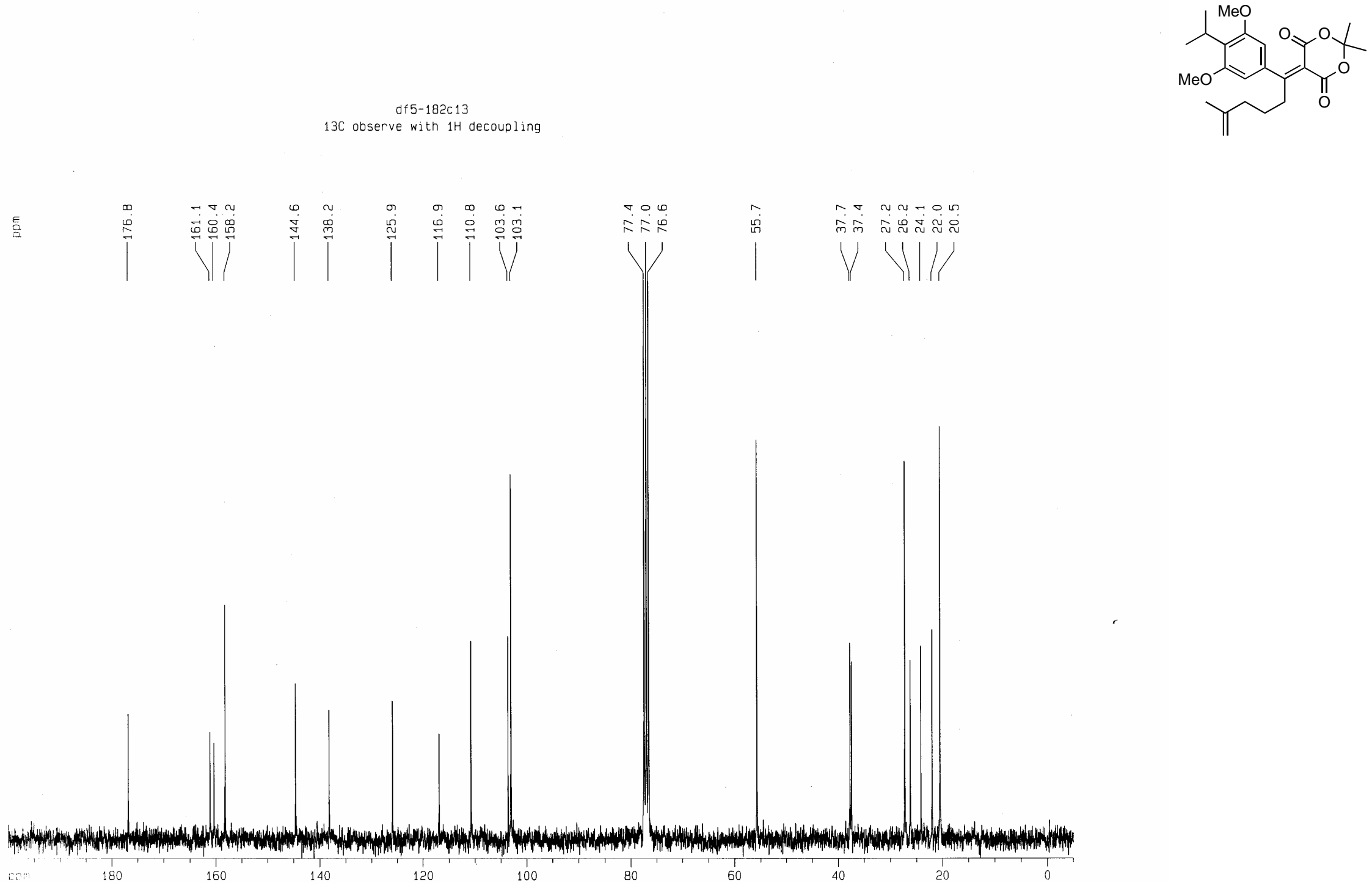

SI-2-15 
(士)-5-[1-(4-isopropyl-3,5-dimethoxyphenyl)-1,5-dimethyl-5-hexenyl]-2,2-dimethyl-1,3-dioxane-4,6-dione (4a)

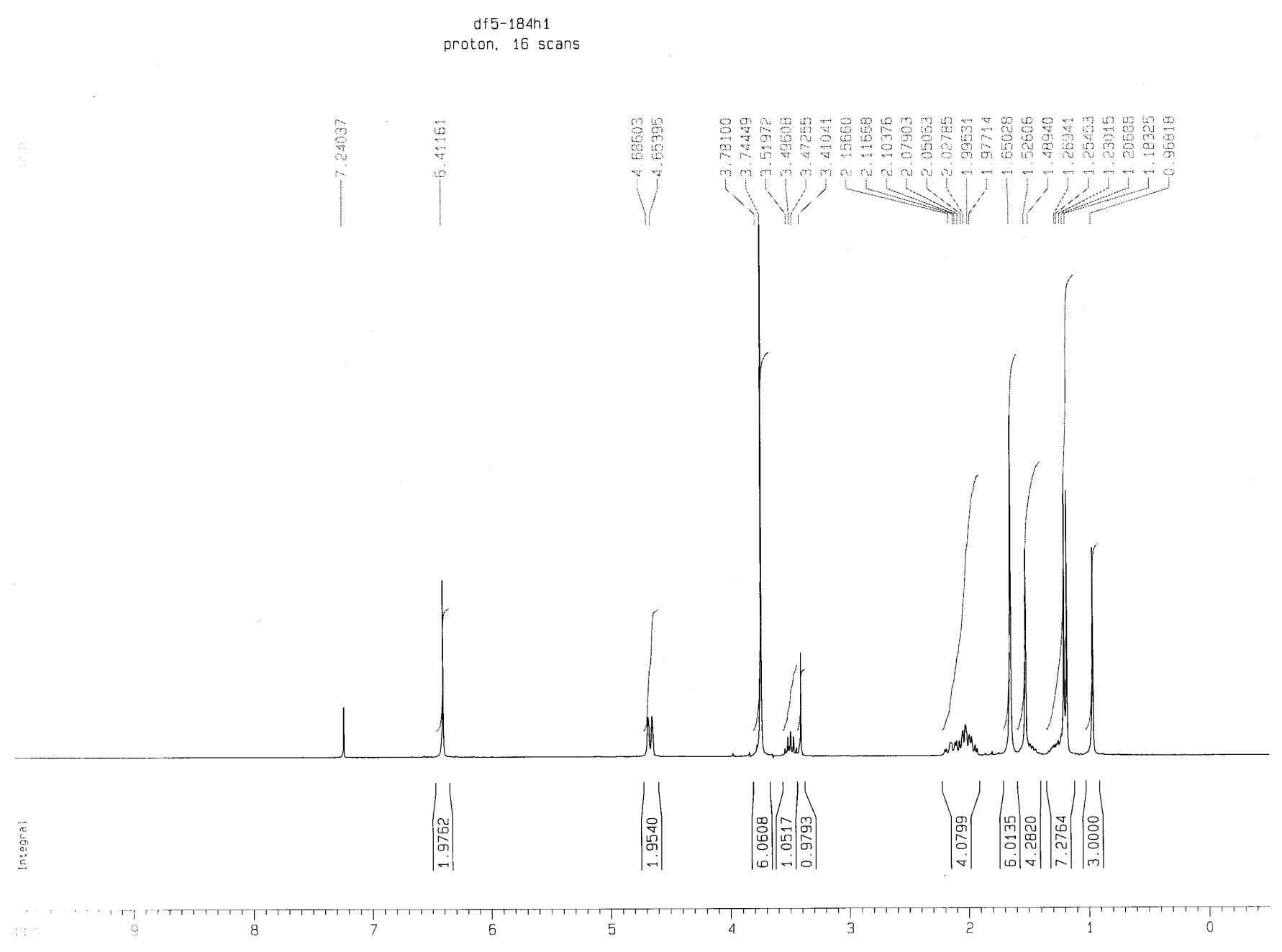

SI-2-16 
(士)-5-[1-(4-isopropyl-3,5-dimethoxyphenyl)-1,5-dimethyl-5-hexenyl]-2,2-dimethyl-1,3-dioxane-4,6-dione (4a)

df5-184c 13

$13 \mathrm{C}$ observe with $1 \mathrm{H}$ decoupling

흠

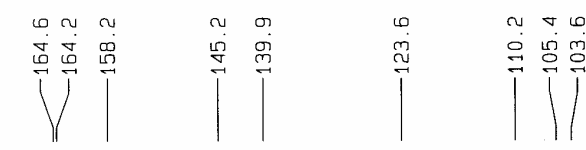

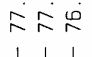

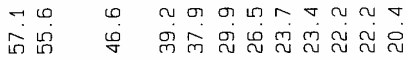

I U UYYK

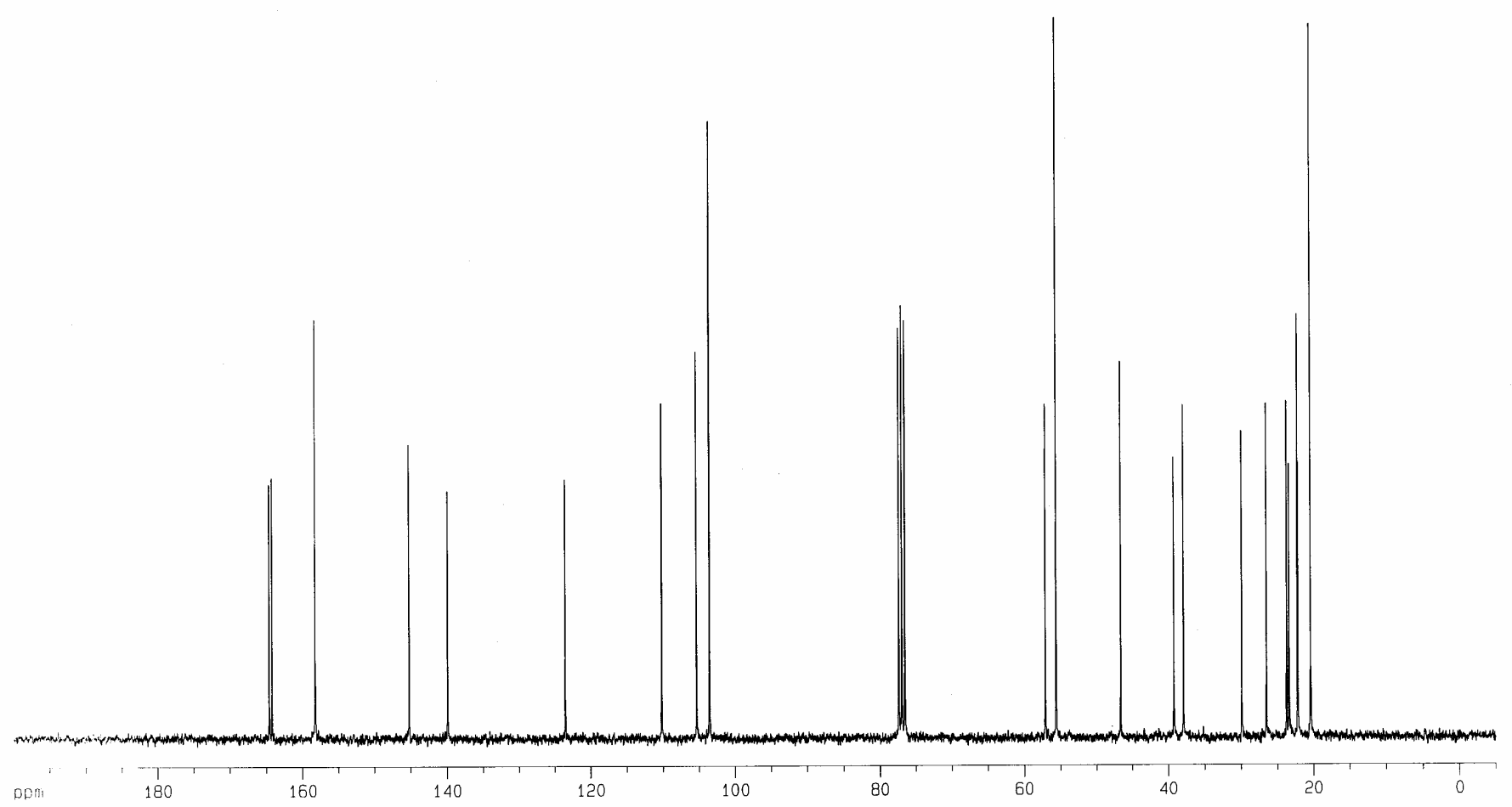




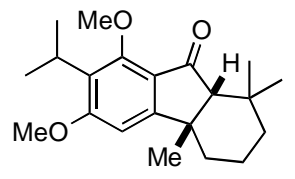

proton, 16 scans

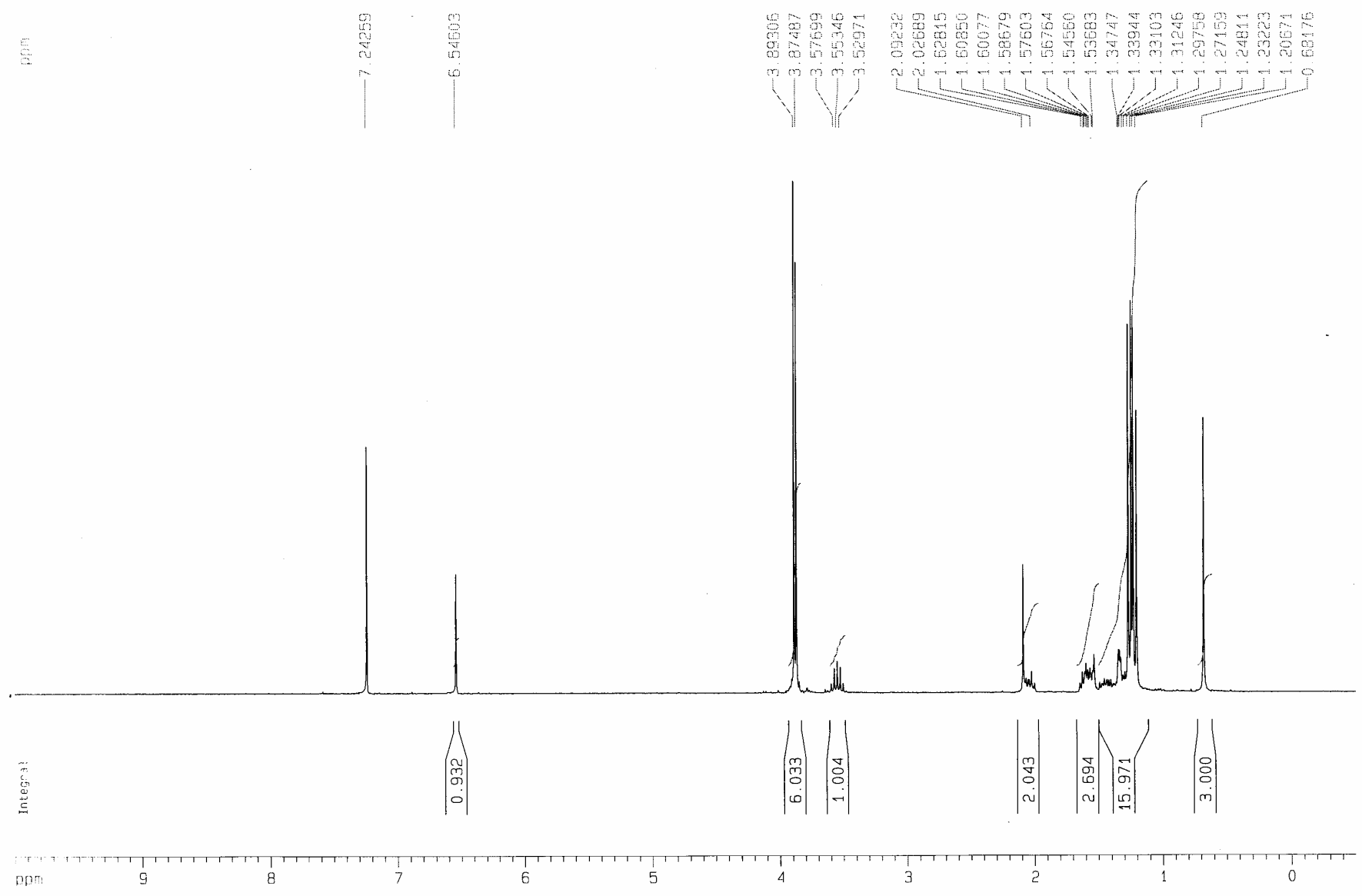


( \pm )-7-Isopropyl-6,8-dimethoxy-1,1,4a-trimethyl-2,3,4,4a,9,9a-hexahydro-1H-9-fluorenone (2a)

Jmod $\mathrm{C}-13$ spectrum with $1 \mathrm{k}$ scans
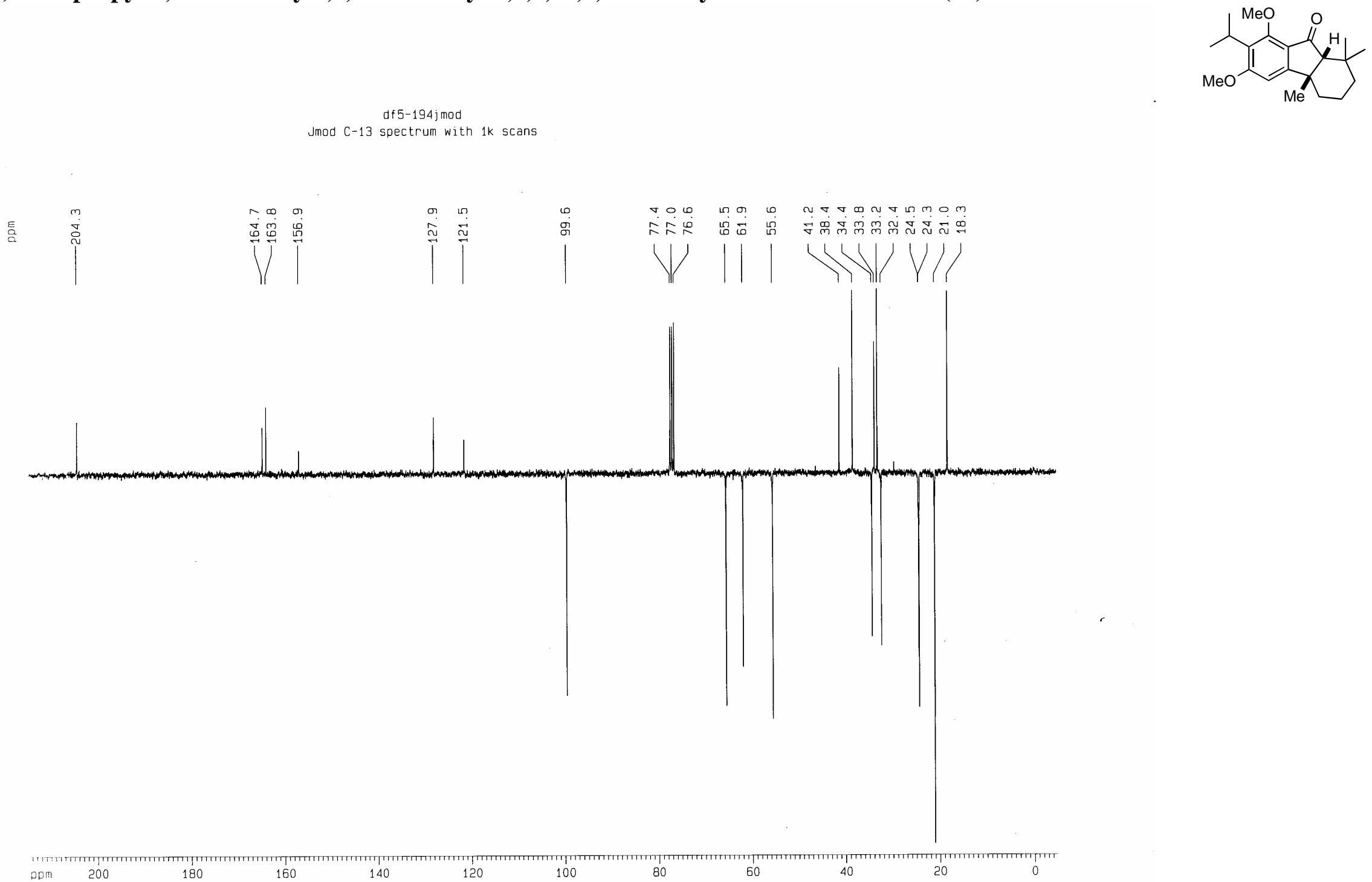
6-Isopropyl-5,7-Dimethoxy-3-Methyl-3-(4-Methyl-3-pentenyl)-1-indanone (11b)

6-Isopropyl-5,7-Dimethoxy-3-Methyl-3-(4-Methyl-4-pentenyl)-1-indanone (11a)

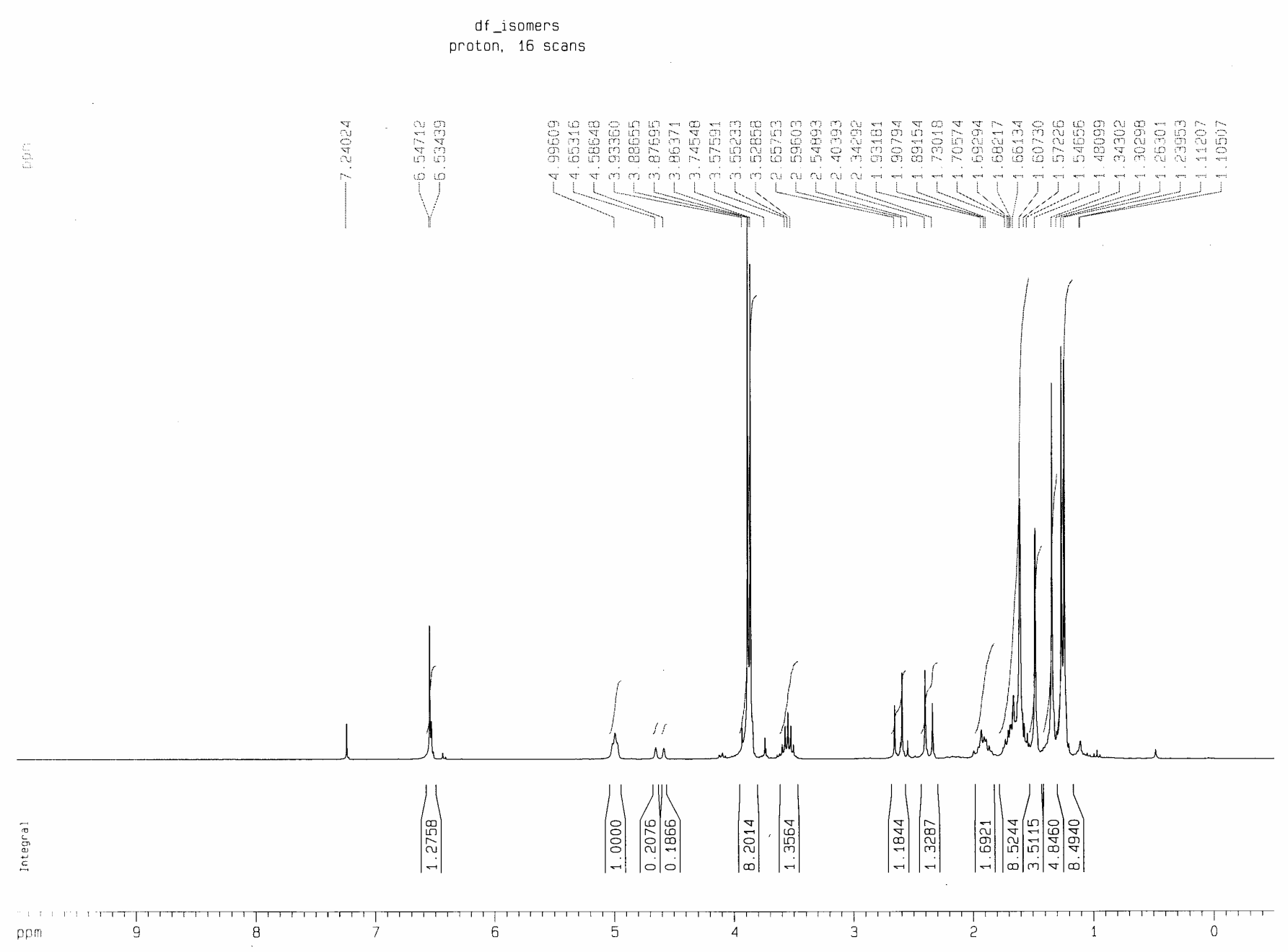

SI-2-20 
6-Isopropyl-5,7-Dimethoxy-3-Methyl-3-(4-Methyl-3-pentenyl)-1-indanone (11b)

6-Isopropyl-5,7-Dimethoxy-3-Methyl-3-(4-Methyl-4-pentenyl)-1-indanone (11a)

df_isomers_jmod

Jmod $\mathrm{C}-13$ spectrum with $1 \mathrm{k}$ scans
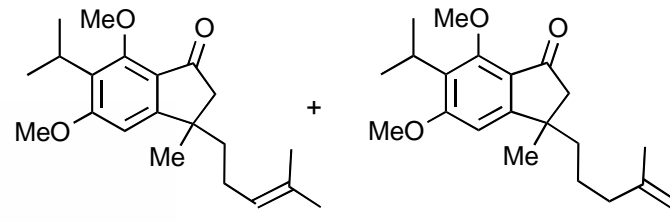
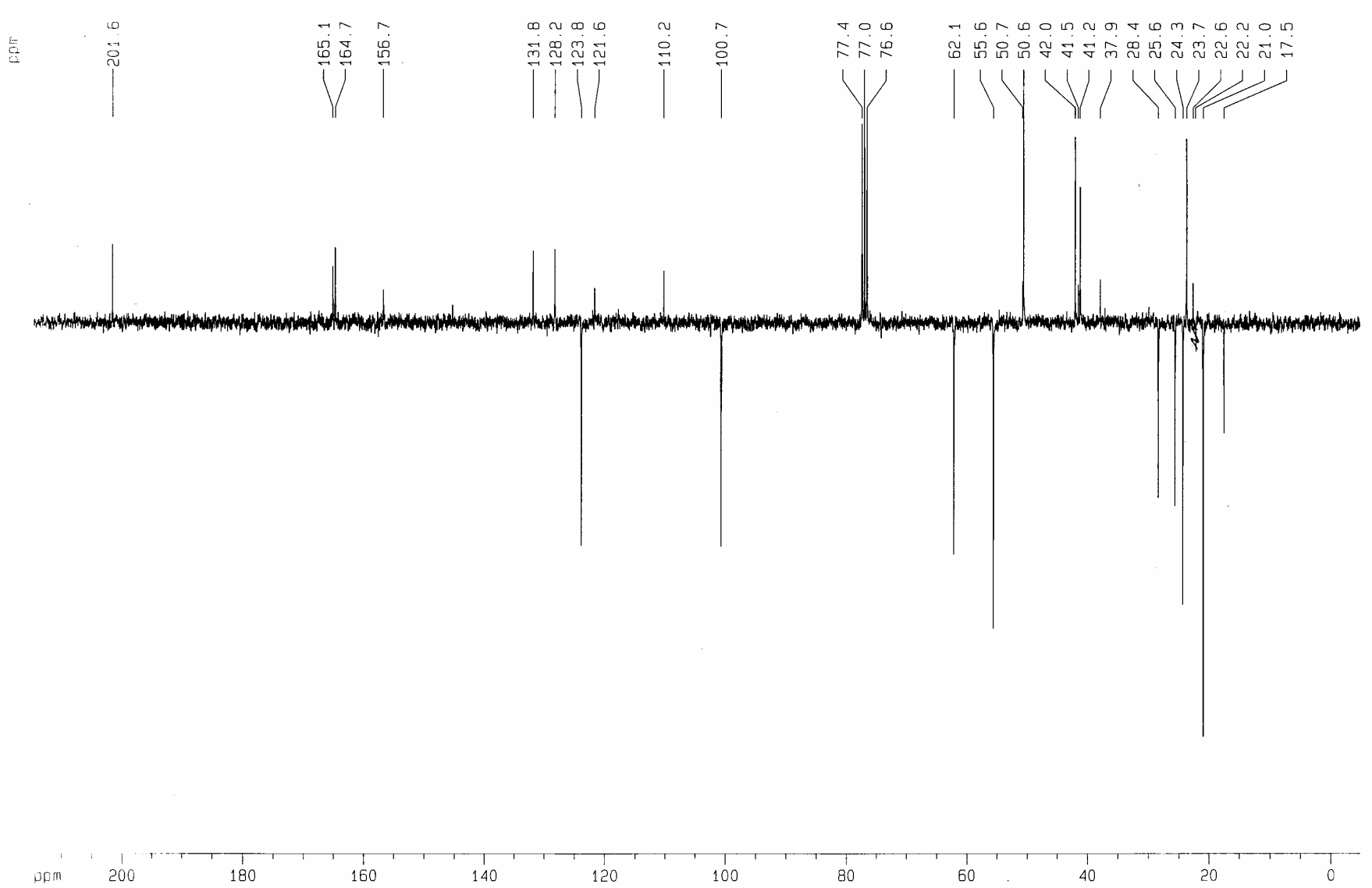

120

80 
(士)-5-[1-(4-Isopropyl-3,5-dimethoxyphenyl)-1,5-dimethyl-4-hexenyl]-2,2-dimethyl-1,3-dioxane-4,6-dione (4b)

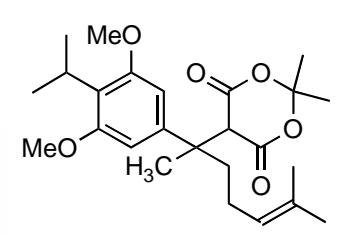

df 5-221h proton, 16 scans

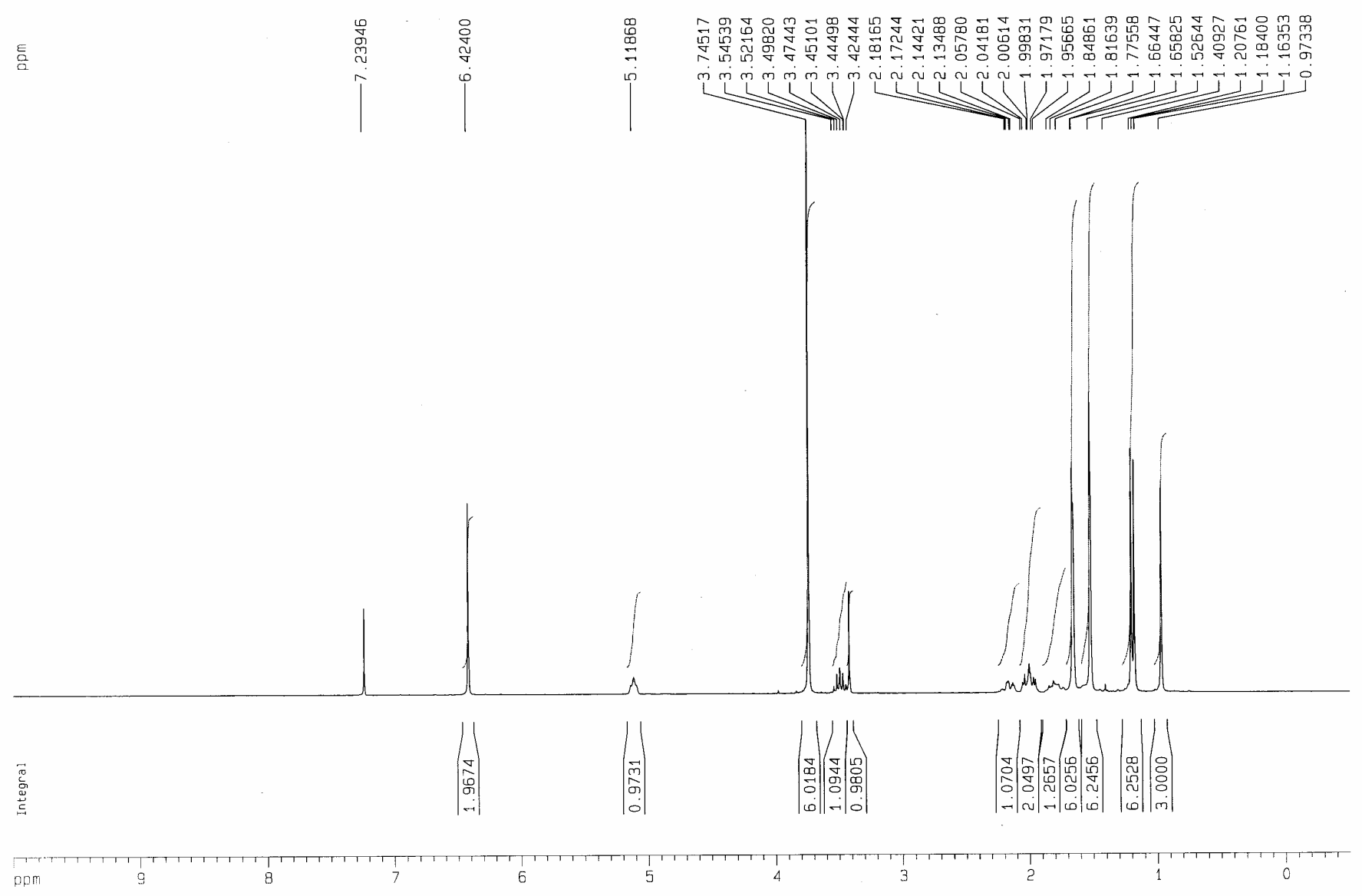


( \pm )-5-[1-(4-Isopropyl-3,5-dimethoxyphenyl)-1,5-dimethyl-4-hexenyl]-2,2-dimethyl-1,3-dioxane-4,6-dione (4b)

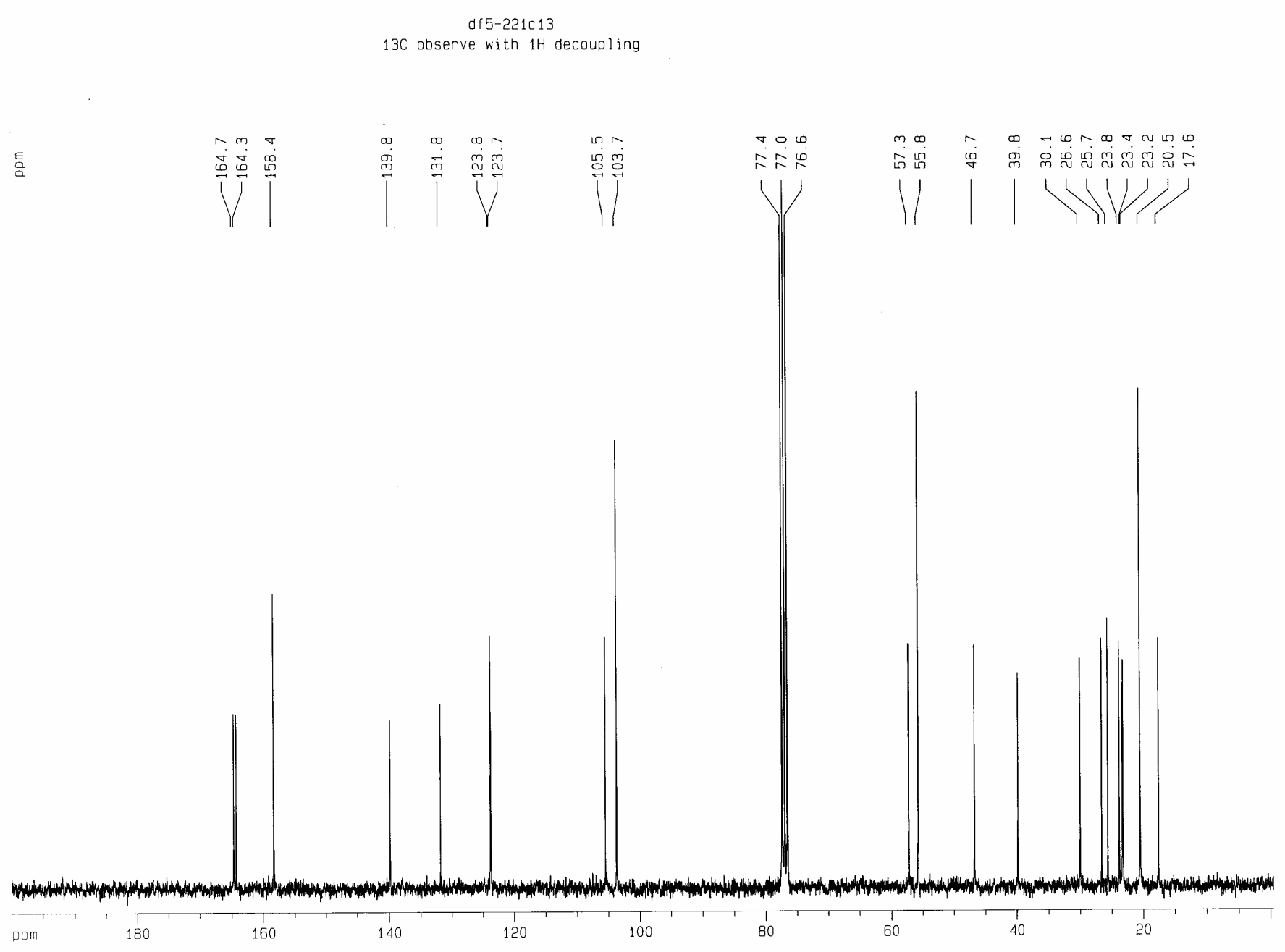

SI-2-23 
( \pm )-5-[1-(4-Isopropyl-3,5-dimethoxyphenyl)-1,4-dimethyl-4-pentenyl]-2,2-dimethyl-1,3-dioxane-4,6-dione (4c)

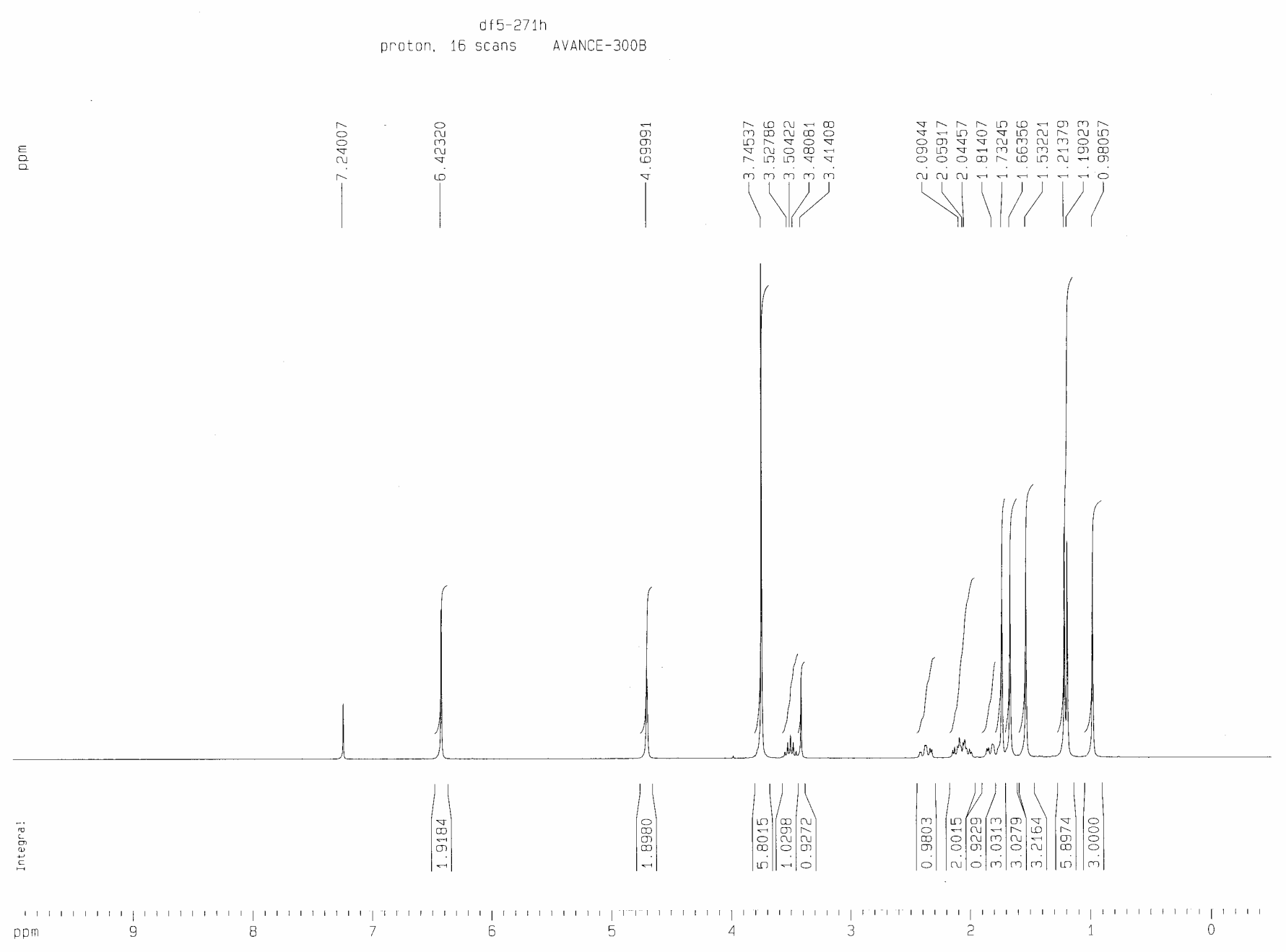

SI-2-24 
(土)-5-[1-(4-Isopropyl-3,5-dimethoxyphenyl)-1,4-dimethyl-4-pentenyl]-2,2-dimethyl-1,3-dioxane-4,6-dione (4c)

df $5-271 \mathrm{jmod}$

C-13 with Decoupling JMOD AVANCE-300B

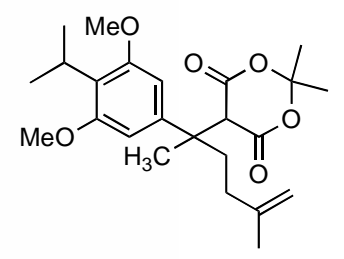

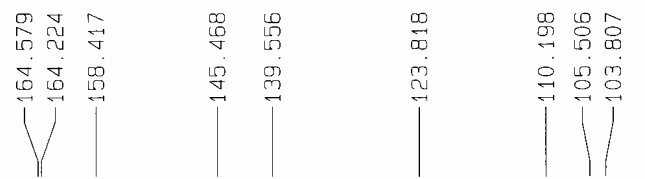
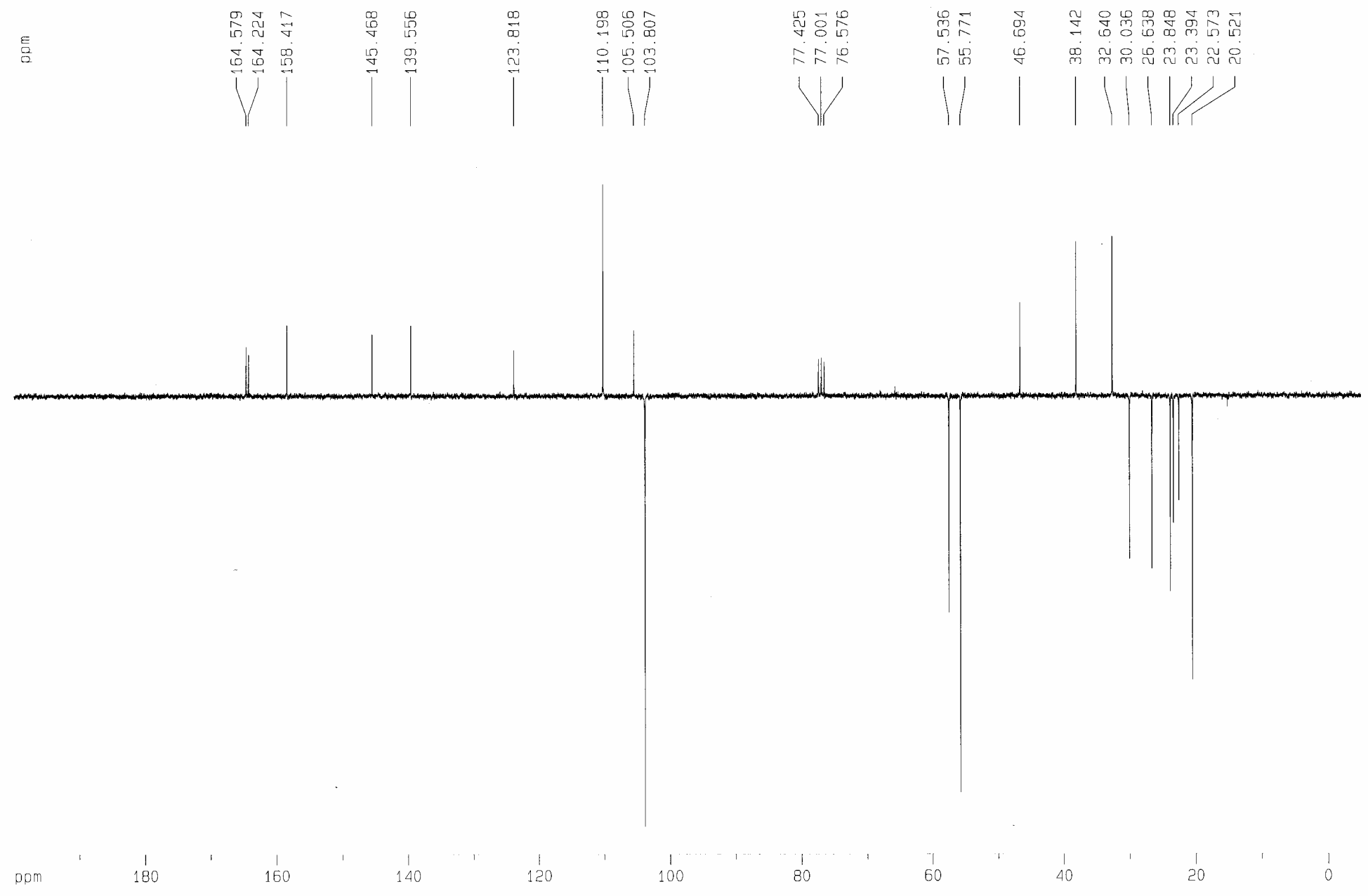
6-Isopropyl-5,7-dimethoxy-1,1,3a-trimethyl-1,2,3,3a,8,8a-hexahydrocyclopenta[a]inden-8-one (2b)

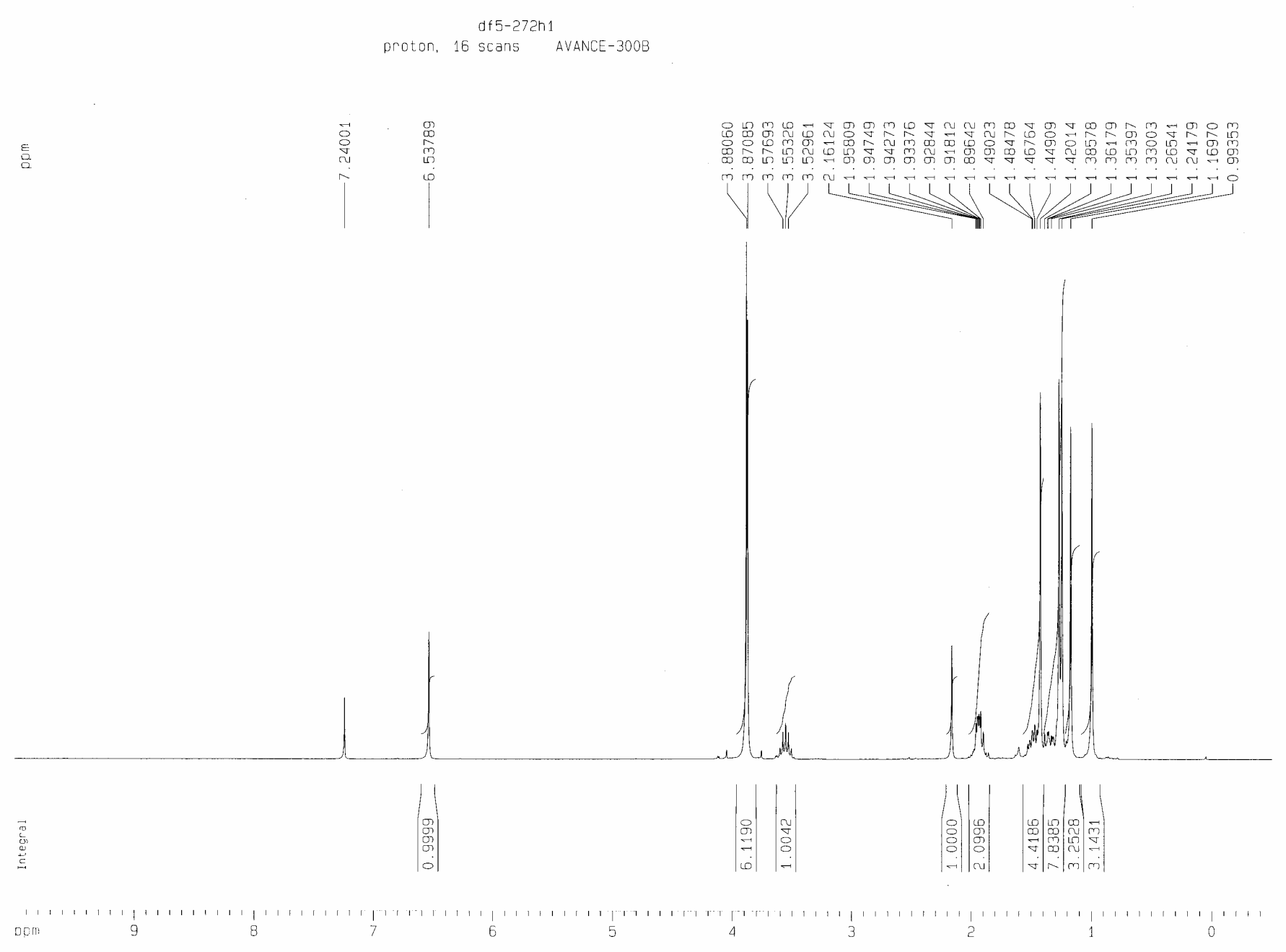


6-Isopropyl-5,7-dimethoxy-1,1,3a-trimethyl-1,2,3,3a,8,8a-hexahydrocyclopenta[a]inden-8-one (2b)

df5-272xst jmod

C-13 with Decoupling JMOD AVANCE-300B

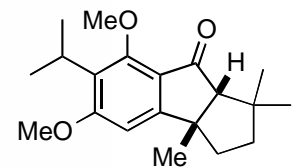

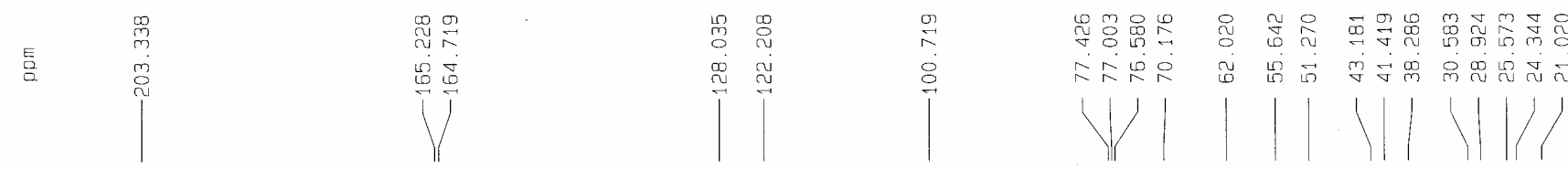

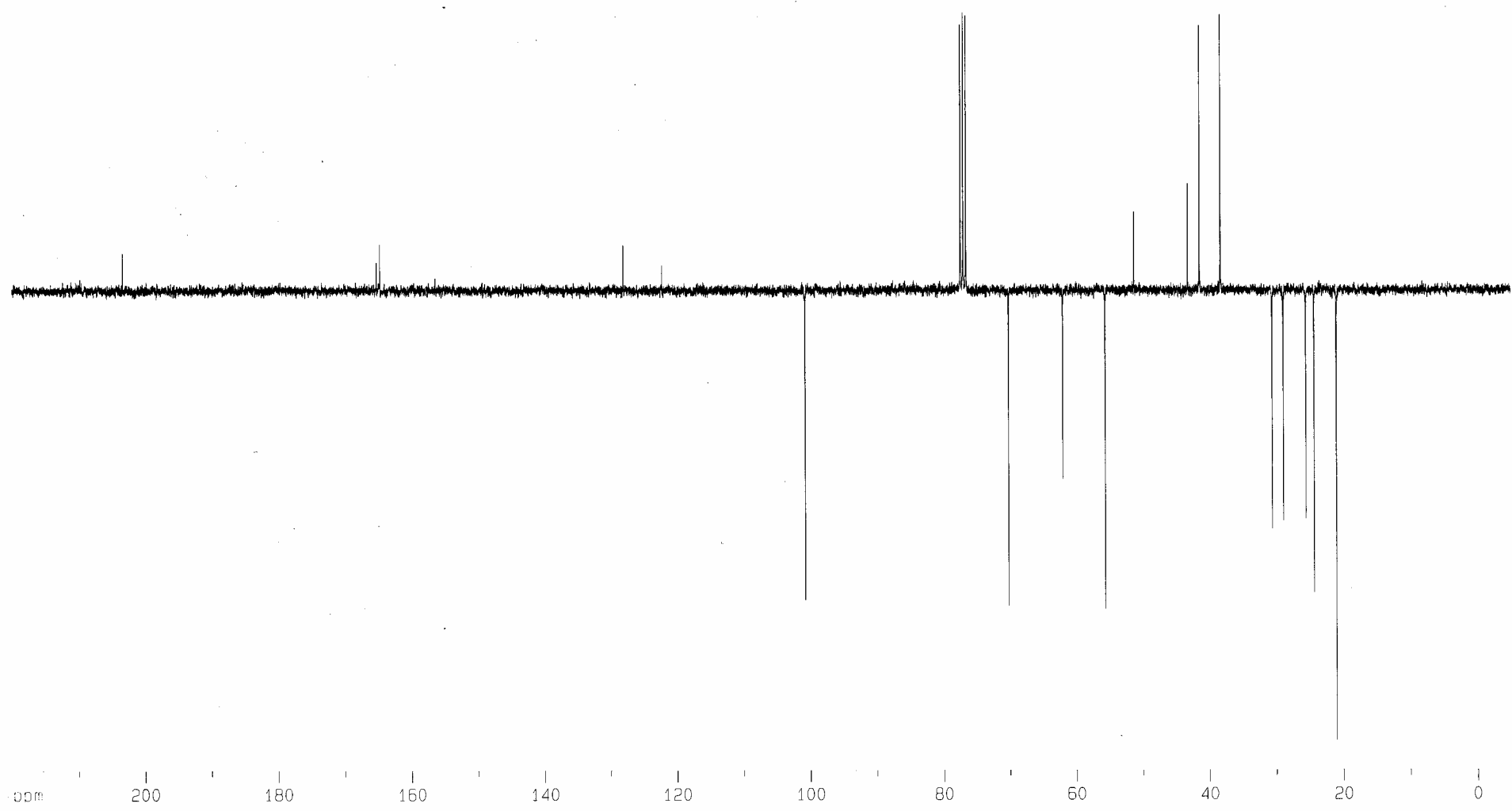


7-Isopropyl-6,8-dimethoxy-2a,5,5-trimethyl-1,2,2a,3,4,5-hexahydro-1-acenaphthylenone (12)

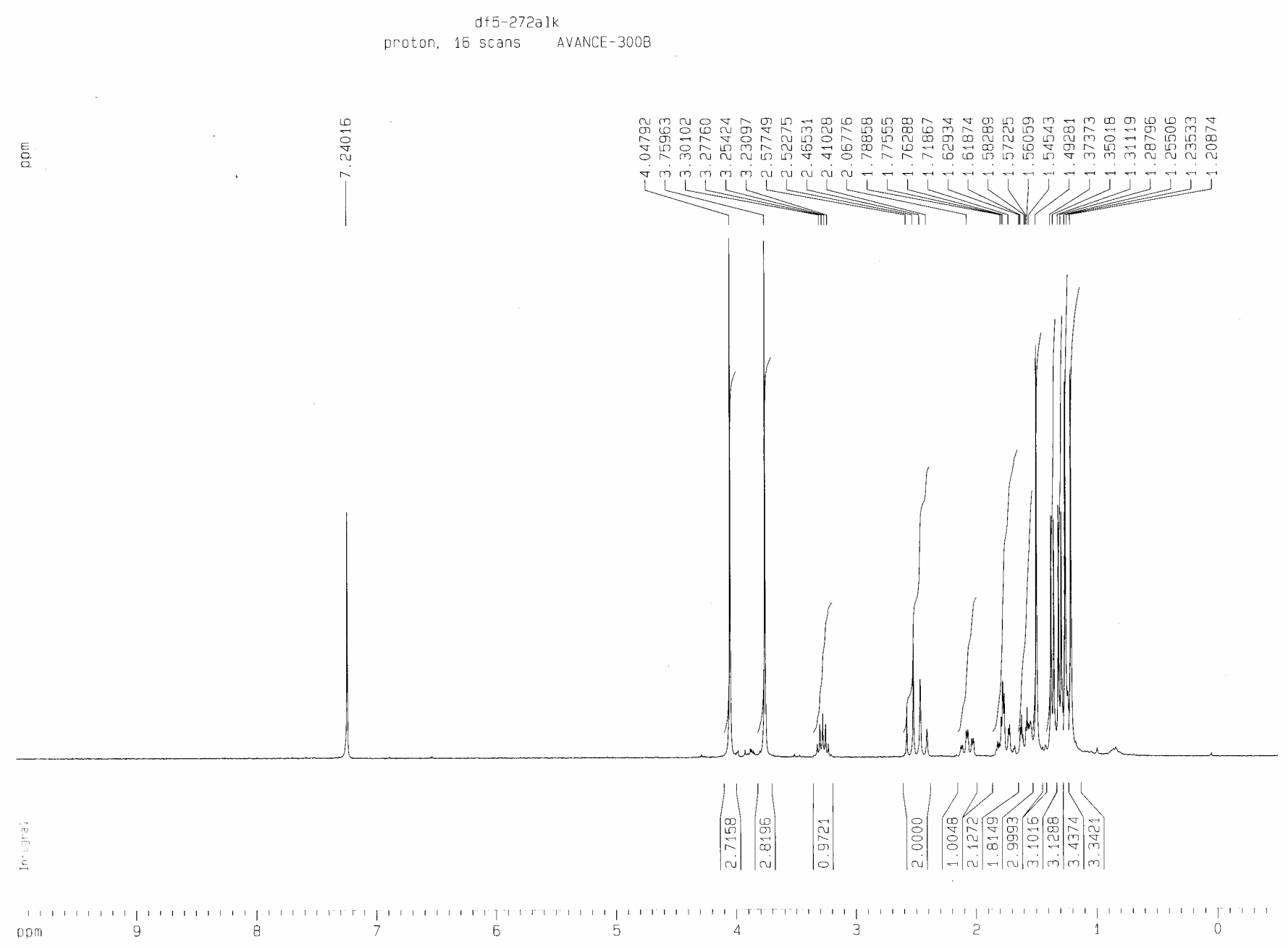




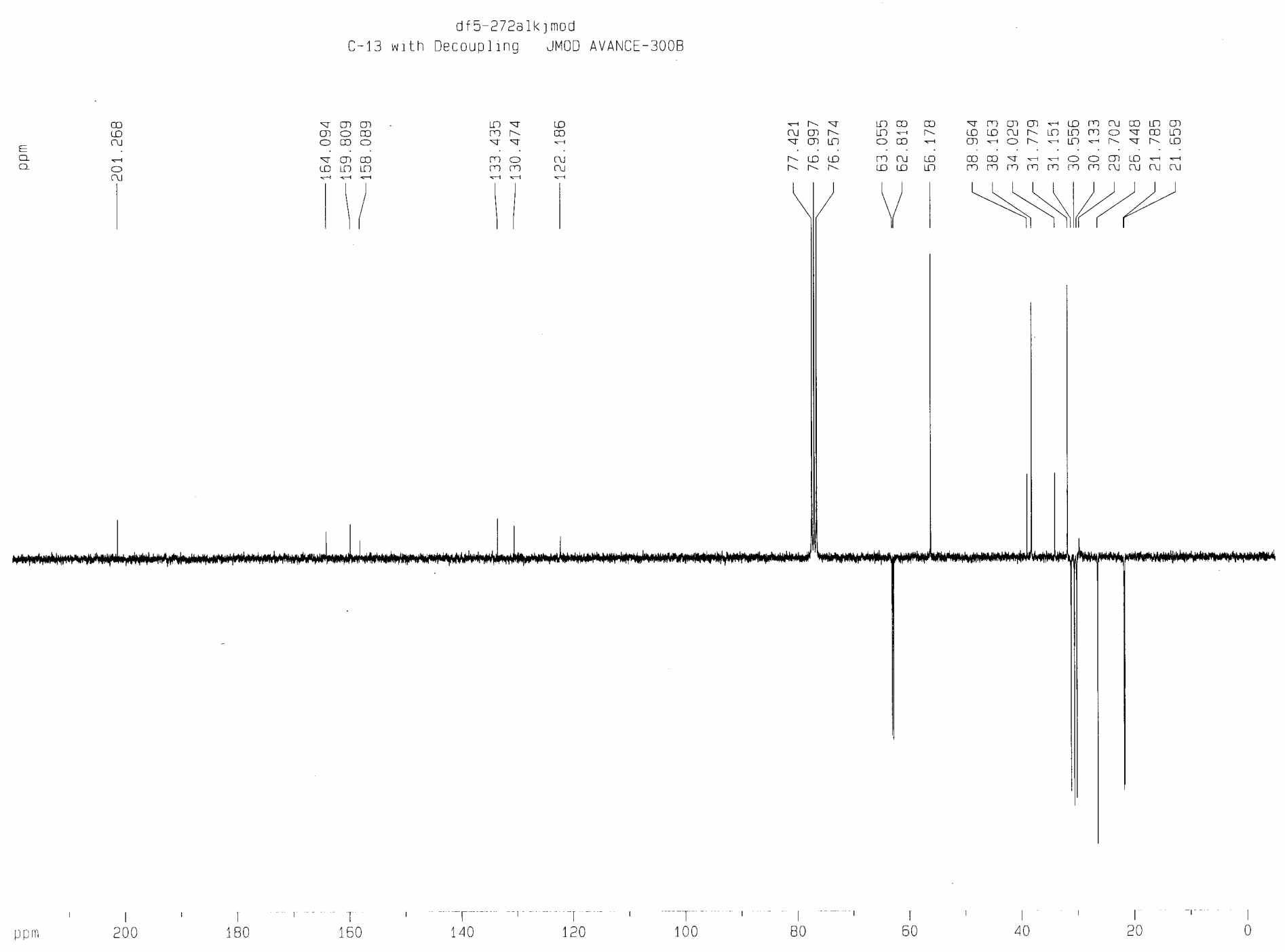


(士)-8-Hydroxy-7-isopropyl-6-dimethoxy-1,1,4a-trimethyl-2,3,4,4a,9,9a-hexahydro-1H-9-fluorenone

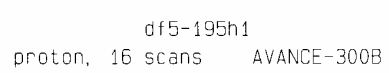
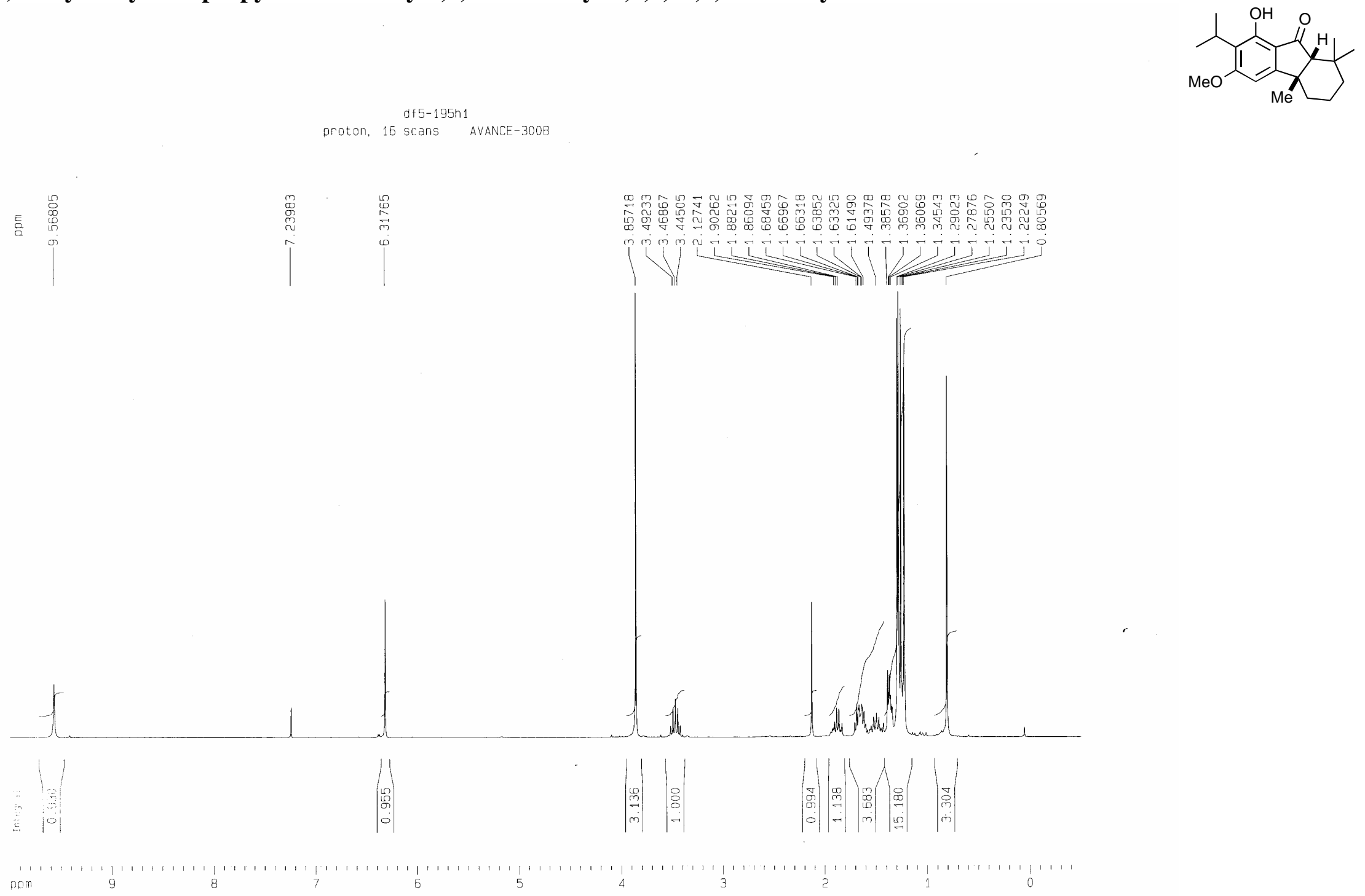
( \pm )-8-Hydroxy-7-isopropyl-6-dimethoxy-1,1,4a-trimethyl-2,3,4,4a,9,9a-hexahydro-1H-9-fluorenone

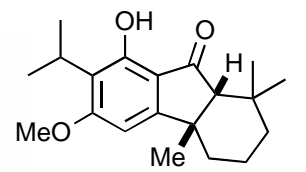

df $5-195$ j mod

C-13 with Decouding JMOD AVANCE-3000

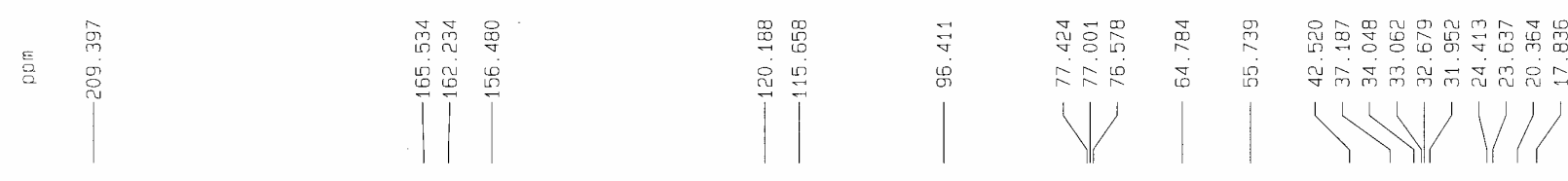

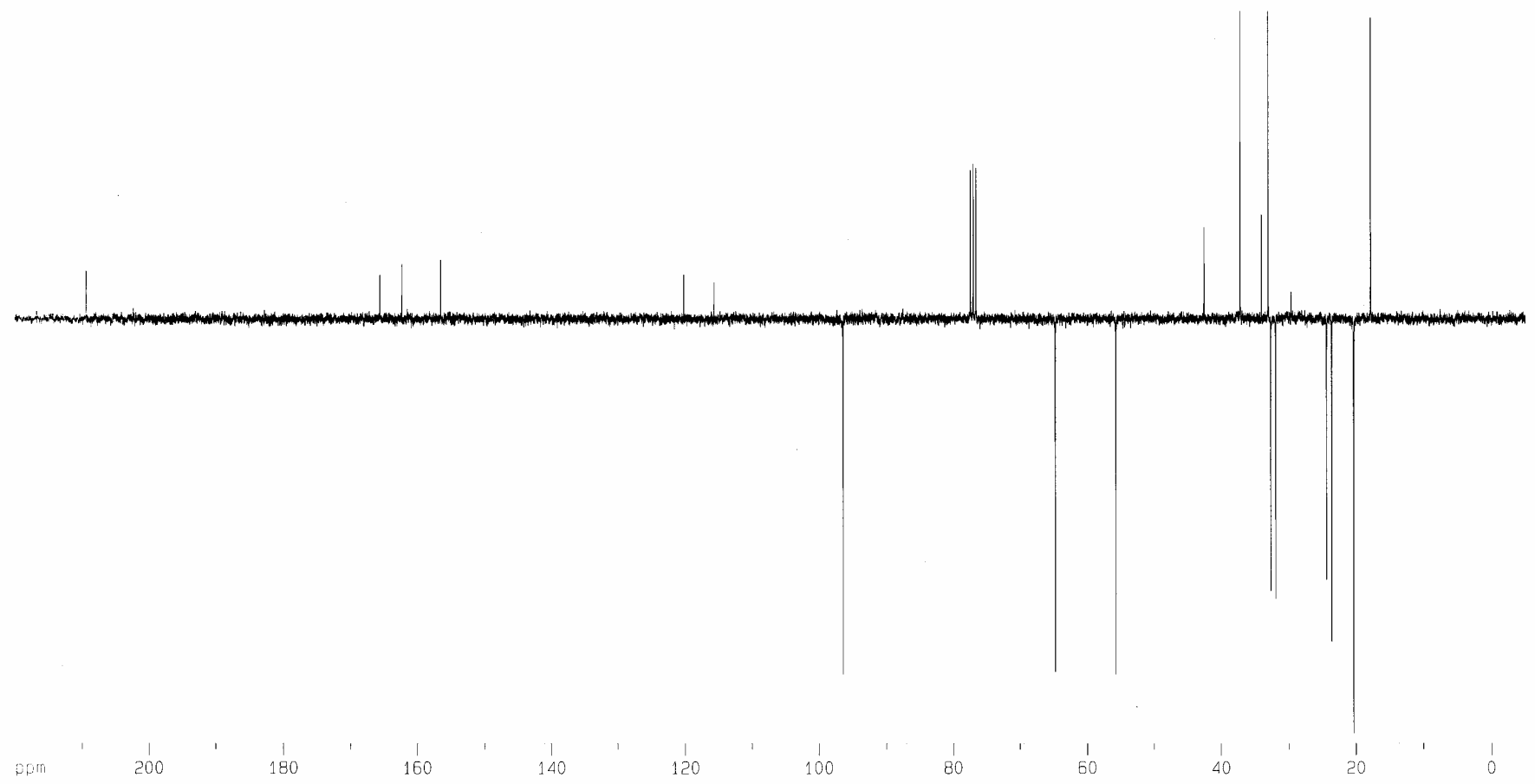


( \pm )-Taiwaniaquinol B

[(土)-5,8-Dihydroxy-7-isopropyl-6-dimethoxy-1,1,4a-trimethyl-2,3,4,4a,9,9a-hexahydro-1H-9-fluorenone] (1)

taiwaniaquinol_b

proton, 16 scans aVANCE-300B
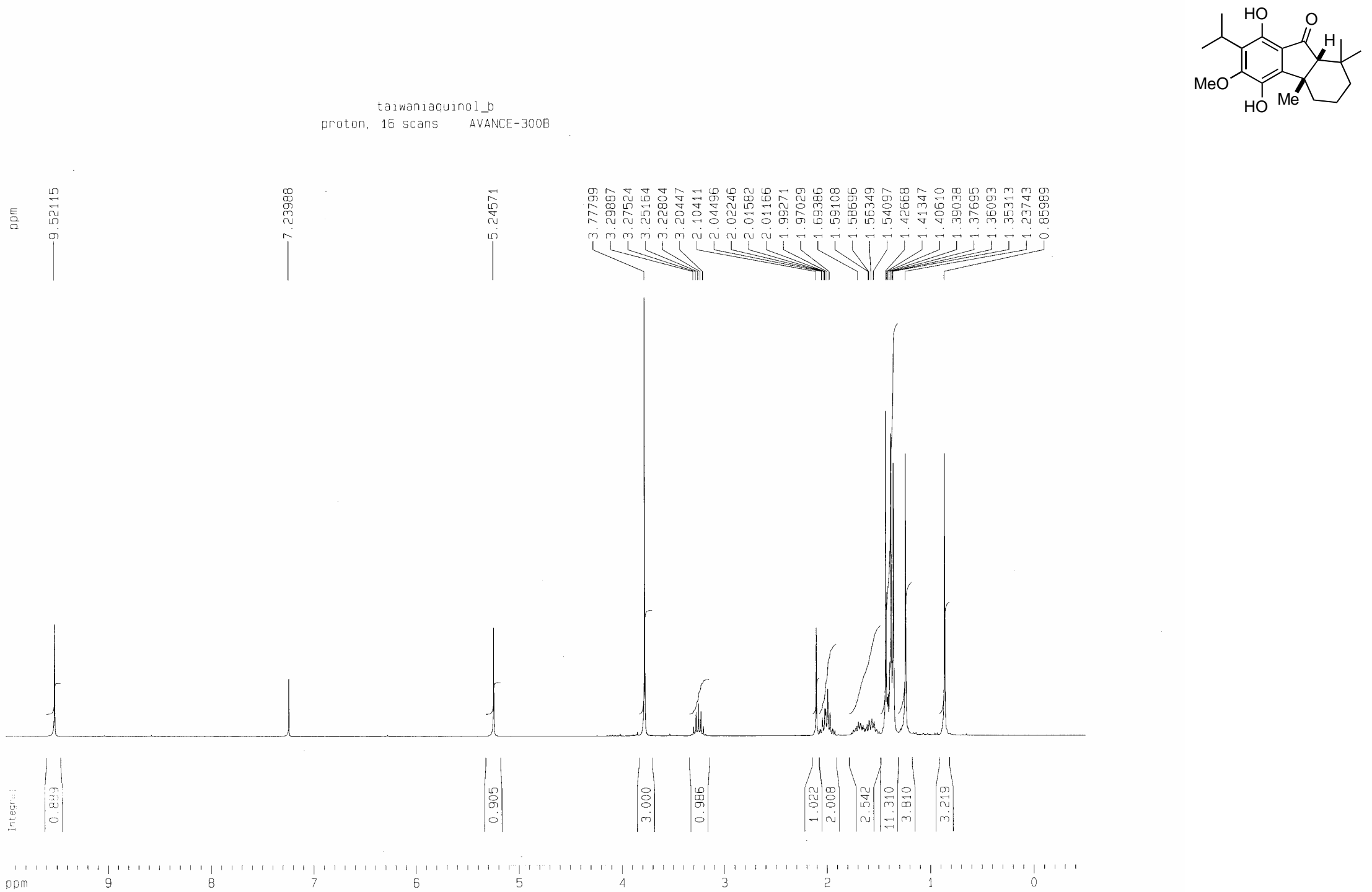

SI-2-32 
( \pm )-Taiwaniaquinol B

[(土)-5,8-Dihydroxy-7-isopropyl-6-dimethoxy-1,1,4a-trimethyl-2,3,4,4a,9,9a-hexahydro-1H-9-fluorenone] (1)

taiwaniaquinol b

C-13 with Decoupling JMOD AVANCE-300B

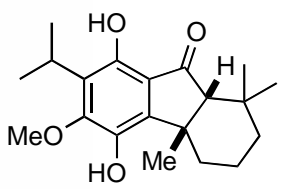

E

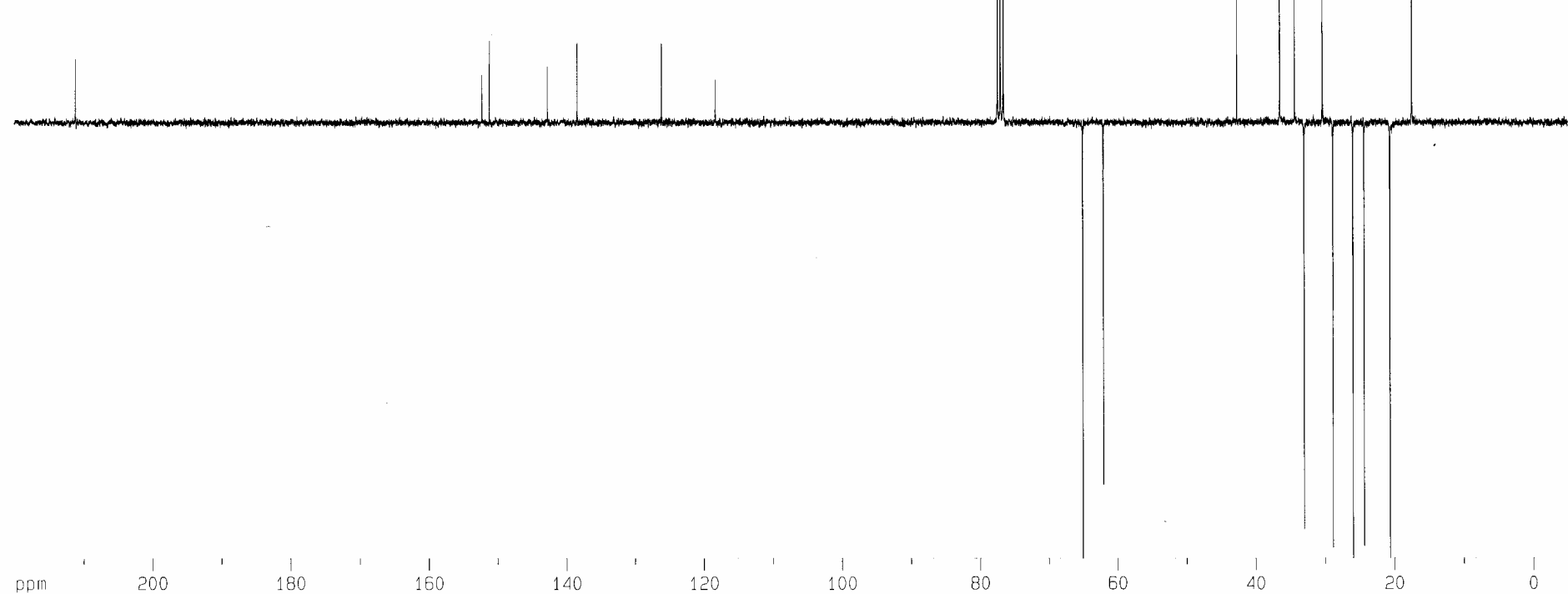

